\title{
The AGN fuelling/feedback cycle in nearby radio galaxies - IV. Molecular gas conditions and jet-ISM interaction in NGC 3100
}

\author{
Ilaria Ruffa ${ }^{\oplus}, 1,2 \star$ Isabella Prandoni ${ }^{\oplus},{ }^{2}$ Timothy A. Davis ${ }^{\bullet},{ }^{1}$ Robert A. Laing, ${ }^{3}$ Rosita Paladino, ${ }^{2}$ \\ Viviana Casasola, ${ }^{2}$ Paola Parma ${ }^{2}$ and Martin Bureau ${ }^{4}$ \\ ${ }^{1}$ Cardiff Hub for Astrophysics Research \& Technology, School of Physics \& Astronomy, Cardiff University, Queens Buildings, The Parade, Cardiff CF24 3AA, \\ $U K$ \\ ${ }^{2}$ INAF, Istituto di Radioastronomia, via P. Gobetti 101, I-40129 Bologna, Italy \\ ${ }^{3}$ Square Kilometre Array Organisation, Jodrell Bank Observatory, Lower Withington, Macclesfield, Cheshire SK11 9DL, UK \\ ${ }^{4}$ Sub-dept. of Astrophysics, Department of Physics, University of Oxford, Denys Wilkinson Building, Keble Road, Oxford OX1 3RH, UK
}

Accepted 2021 November 30. Received 2021 November 15; in original form 2021 July 24

\begin{abstract}
This is the fourth paper of a series investigating the AGN fuelling/feedback processes in a sample of 11 nearby low-excitation radio galaxies (LERGs). In this paper, we present follow-up Atacama Large Millimeter/submillimeter Array (ALMA) observations of one source, NGC 3100, targeting the ${ }^{12} \mathrm{CO}(1-0),{ }^{12} \mathrm{CO}(3-2), \mathrm{HCO}^{+}(4-3), \mathrm{SiO}(3-2)$, and $\mathrm{HNCO}(6-5)$ molecular transitions. ${ }^{12} \mathrm{CO}(1-0)$ and ${ }^{12} \mathrm{CO}(3-2)$ lines are nicely detected and complement our previous ${ }^{12} \mathrm{CO}(2-1)$ data. By comparing the relative strength of these three $\mathrm{CO}$ transitions, we find extreme gas excitation conditions (i.e. $T_{\mathrm{ex}} \gtrsim 50 \mathrm{~K}$ ) in regions that are spatially correlated with the radio lobes, supporting the case for a jet-ISM interaction. An accurate study of the CO kinematics demonstrates that although the bulk of the gas is regularly rotating, two distinct non-rotational kinematic components can be identified in the inner gas regions: one can be associated to inflow/outflow streaming motions induced by a two-armed spiral perturbation; the second one is consistent with a jet-induced outflow with $v_{\max } \approx 200 \mathrm{~km} \mathrm{~s}^{-1}$ and $\dot{M} \lesssim 0.12 \mathrm{M}_{\odot} \mathrm{yr}^{-1}$. These values indicate that the jet-CO coupling ongoing in NGC 3100 is only mildly affecting the gas kinematics, as opposed to what expected from existing simulations and other observational studies of (sub-)kpc scale jet-cold gas interactions. $\mathrm{HCO}^{+}(4-3)$ emission is tentatively detected in a small area adjacent to the base of the northern radio lobe, possibly tracing a region of jet-induced gas compression. The $\mathrm{SiO}(3-2)$ and $\mathrm{HNCO}(6-5)$ shock tracers are undetected: this - along with the tentative $\mathrm{HCO}^{+}(4-3)$ detection - may be consistent with a deficiency of very dense (i.e. $n_{\text {crit }}>10^{6} \mathrm{~cm}^{-3}$ ) cold gas in the central regions of NGC 3100 .
\end{abstract}

Key words: galaxies: active - galaxies: elliptical and lenticular, cD - galaxies: individual: NGC3100 - galaxies: ISM-galaxies: jets.

\section{INTRODUCTION}

Both theoretical and observational evidence increasingly suggest that the energy released by feedback processes associated with active galactic nuclei (AGN) is a fundamental component in the evolution of their host galaxies (e.g. Combes 2017; Choi et al. 2018; Pillepich et al. 2019). The complexity of these phenomena, however, makes many of their key aspects still unclear (e.g. Wylezalek \& Morganti 2018; Harrison et al. 2021).

AGN feedback is currently believed to operate in two (nonexclusive) main modes: radiative (or quasar) mode and kinetic (or jet) mode (e.g. Morganti 2017). The former is considered to be dominant in radiatively efficient (quasar- or Seyfert-like) AGN accreting matter at high rates $\left(\gtrsim 0.01 \dot{M}_{\text {Edd }}\right.$, where $\dot{M}_{\text {Edd }}$ is the Eddington accretion rate). ${ }^{1}$ In these objects, the radiation pressure generated by the

\footnotetext{
^E-mail: ruffai@ cardiff.ac.uk

${ }^{1} \dot{M}_{\mathrm{Edd}}=\frac{4 \pi G M_{\mathrm{SMBH}} m_{\mathrm{p}}}{\varepsilon c \sigma_{\mathrm{T}}}$, where $G$ is the gravitational constant, $M_{\mathrm{SMBH}}$ is
}

accretion process can drive powerful outflows (the so-called AGN winds) that are potentially able to expel large quantities of matter from the nuclear regions (e.g. King \& Nixon 2015; Wagner et al. 2016; Bieri et al. 2017; Ishibashi, Fabian \& Maiolino 2018). Kinetic feedback is instead dominant in AGN accreting matter at low rates ( $\ll 0.01 \dot{M}_{\text {Edd }}$; e.g. Cielo et al. 2018), whereby the bulk of the energy generated from the accretion process is channelled into collimated outflows of non-thermal plasma (i.e. the radio jets).

The impact that kinetic AGN feedback can have on large (i.e. tens of $\mathrm{kpc}$ ) scales is well established. Expanding radio jets have been often observed to inflate cavities through the hot X-rayemitting atmospheres of galaxies, groups, and clusters, heating the surroundings, balancing radiative losses, and preventing the gas from further cooling (see e.g. McNamara \& Nulsen 2012, for a review). In the past decade, however, jet-mode feedback has been increasingly observed to alter the distribution, kinematics, and physics of the surrounding gaseous medium also on (sub-)kpc scales (e.g. Combes

$\varepsilon$ is the accretion efficiency, $c$ is the speed of light, and $\sigma_{\mathrm{T}}$ is the cross-section for Thomson scattering. 
et al. 2013; García-Burillo et al. 2014; Morganti et al. 2015; Mahony et al. 2016; Murthy et al. 2019; Zovaro et al. 2019; Santoro et al. 2020; Venturi et al. 2021, see also Veilleux et al. 2020, for a recent review on this subject). Observations find support in three-dimensional (3D) hydrodynamical simulations (e.g. Wagner, Bicknell \& Umemura 2012; Wagner et al. 2016; Mukherjee et al. 2016, 2018a,b) showing that young and compact radio jets expanding through the surrounding layers of matter can produce turbulent cocoons of shocked gas that can be accelerated up to $1000 \mathrm{~km} \mathrm{~s}^{-1}$ over a wide range of directions. The extent of jet-induced perturbations has been predicted to vary depending on the radio jet power, relative jet-gas orientation, and age of the radio source. Large samples of objects covering wide ranges of jet ages, radio powers, and jet-ISM geometric configurations are then required to gain a better understanding of the incidence and properties of jet-ISM interactions. This is essential information to fully assess the role of jet-induced feedback in galaxy evolution (e.g. Morganti, Oosterloo \& Tadhunter 2021).

Molecular line observations are powerful tools to investigate the physical conditions of the gas in various environments, as different molecules, isotopologues, and transitions of the same species trace different gas components within the same galaxy. The most used molecular gas tracer is $\mathrm{CO}$ : it is the most abundant molecule after $\mathrm{H}_{2}$ and its low-excitation temperature (i.e. $T_{\mathrm{ex}}=5.53 \mathrm{~K}$ for the ground $J=1 \rightarrow 0$ rotational transition; e.g. Carilli \& Walter 2013) makes it easily excited even at low temperatures. The critical density (i.e. the density at which collisional excitation balances spontaneous radiative de-excitation) for the $\mathrm{CO}(1-0)$ transition is $n_{\text {crit }} \approx 2200 \mathrm{~cm}^{-3}$. Higher$J$ transitions require rapidly increasing densities and temperatures to be excited (i.e. $n_{\text {crit }} \propto J^{3}$ and $T_{\text {ex }} \propto J^{2}$; see e.g. Bolatto, Wolfire \& Leroy 2013); therefore, ratios of multiple CO lines can be used to probe the excitation temperature and density of molecular gas (e.g. Kamenetzky, Rangwala \& Glenn 2017; Saito et al. 2017; Kamenetzky, Privon \& Narayanan 2018). Ratios of ${ }^{12} \mathrm{CO}$ and ${ }^{13} \mathrm{CO}$ isotopologues can also provide estimates of the gas optical depth, as ${ }^{12} \mathrm{CO}$ emission is generally optically thick, while ${ }^{13} \mathrm{CO}$ is mostly (but not totally; see e.g. Cormier et al. 2018) optically thin over large areas (e.g. Jiménez-Donaire et al. 2017). The SiO molecule is commonly considered a good tracer of shocks. Indeed, silicon particles are mostly found in the core of dust grains and can be released only in grain sputtering or evaporation caused by high-velocity shocks. Once Si-bearing material is in the gas phase, it can react with molecular oxygen to form $\mathrm{SiO}$ (Schilke et al. 1997), which can then be used to trace regions of fast shocks ( $v>50 \mathrm{~km} \mathrm{~s}^{-1}$; e.g. Usero et al. 2006). Growing observational evidence suggests that the HNCO molecule is a particularly good tracer of slow $\left(v<20 \mathrm{~km} \mathrm{~s}^{-1}\right)$ shocks: its presence in the outer layers of dust grains means that it is easily sublimated, and it remains detectable only in weakly shocked regions (e.g. Kelly et al. 2017). $\mathrm{HCO}^{+}$, along with CS, $\mathrm{HCN}$, and $\mathrm{HNC}$, is the most used tracer of the dense $\left(n_{\text {crit }}>10^{4} \mathrm{~cm}^{-3}\right)$ cold gas component (e.g. Gao \& Solomon 2004; Costagliola et al. 2011; García-Burillo et al. 2014). The abundance of these molecules, relative to $\mathrm{CO}$, is commonly used to investigate the density conditions of the cold gas (e.g. Usero et al. 2015; Bigiel et al. 2016; Topal et al. 2016). A multi-species approach is therefore key to properly characterize the impact of jet-induced feedback on the physics of the surrounding cold gas.

Despite their relevance, detailed spatially resolved studies of jetISM interactions using multiple molecular transitions have been carried out so far only for a limited number of objects, mostly nearby (Seyfert-like) galaxies hosting (sub-)kpc scale jets (see e.g. Alatalo et al. 2013; Combes et al. 2013; García-Burillo et al. 2014; Feruglio et al. 2015; Oosterloo et al. 2017, and references therein).
Table 1. General properties of NGC 3100 .

\begin{tabular}{lcc}
\hline ID & Parameter & Value \\
\hline$(1)$ & Type & S0 \\
$(2)$ & RA (J2000) & $10^{\mathrm{h}} 00^{\mathrm{m}} 40^{\mathrm{s}} .8$ \\
$(3)$ & DEC (J2000) & $-31^{\circ} 39^{\prime} 52^{\prime \prime} .3$ \\
$(4)$ & Redshift & 0.0088 \\
$(5)$ & $\mathrm{D}_{\mathrm{L}}(\mathrm{Mpc})$ & 38.0 \\
$(6)$ & Radio Source & PKS $0958-314$ \\
$(7)$ & $\log P_{1.4 \mathrm{GHz}}\left(\mathrm{W} \mathrm{Hz}^{-1}\right)$ & 23.0 \\
$(8)$ & $v_{\text {opt }}\left(\mathrm{km} \mathrm{s}^{-1}\right)$ & $2629 \pm 20$ \\
\hline
\end{tabular}

Note. Rows: (1) Morphological type of the galaxy from the HyperLeda database (http://leda.univ-lyon1.fr). (2) and (3) Galaxy sky coordinates from Petrov et al. (2005). (4) Galaxy redshift from Dopita et al. (2015). (5) Luminosity distance derived from the tabulated redshift and assuming the cosmology stated in Section 1. (6) Name of the radio source. (7) Radio power at $1.4 \mathrm{GHz}$ derived from the flux density given in Brown et al. (2011), including all the radio emission associated with the source. (8) Optical stellar velocity from Kerr \& Lynden-Bell (1986).

These few existing studies show that line ratios are drastically modified in the (vicinity of) regions where kinetic AGN feedback is in action, implying great differences between the physical conditions of the quiescent and interacting gas: the latter is usually clumpy, with much higher temperatures and densities. Signs of compression and fragmentation in the molecular gas distribution have also been observed and attributed to the driving mechanism of the interaction.

Our aim is to expand this kind of studies to a specific class of low-power radio galaxies, known as low-excitation radio galaxies (LERG). LERGs are, by number, the dominant radio galaxy population in the local Universe (e.g. Hardcastle, Evans \& Croston 2007), predominantly hosted by red, massive $\left(M_{*} \geq 10^{11} \mathrm{M}_{\odot}\right)$ early-type galaxies (ETGs; e.g. Best \& Heckman 2012). These objects mostly have Fanaroff-Riley type I radio morphologies (FRI; Fanaroff \& Riley 1974) and low or moderate radio luminosities $\left(P_{1.4 \mathrm{GHz}}<10^{25} \mathrm{~W} \mathrm{~Hz}{ }^{-1}\right)$. The central super-massive black holes (SMBHs) in LERGs accrete matter at low rates $\left(\ll 0.01 \dot{M}_{\text {Edd }}\right)$ and produce almost exclusively kinetic feedback. Few detailed, spatially resolved studies of LERGs have yet been made, so their triggering mechanisms and associated AGN feedback processes are still poorly understood (e.g. Hardcastle 2018): investigating the nature of LERGs is crucial to shed light on the mechanisms determining the observed properties of massive nearby ETGs.

We are carrying out the first, extensive, spatially resolved, multiphase study of a small but complete sample of 11 LERGs in the southern sky. In this paper, we present follow-up ALMA observations of one object, NGC 3100 , targeting ${ }^{12} \mathrm{CO}(1-0)$ and ${ }^{12} \mathrm{CO}(3-$ 2) transitions, along with $\mathrm{HCO}^{+}(4-3), \mathrm{SiO}(3-2)$, and $\mathrm{HNCO}(6-5)$ lines. Coupling these new data with previous ${ }^{12} \mathrm{CO}(2-1)$ and radio continuum observations, we primarily aim at carrying out an analysis of the molecular gas physics and jet-CO interplay in this object.

The paper is structured as follows. In Section 2, we briefly present the overall project and the target of this study. In Section 3, we describe ALMA observations and the data reduction. The data analysis is presented in Section 4. We discuss the results in Section 5 before summarizing and concluding in Section 6.

Throughout this work, we assume a standard $\Lambda$ CDM cosmology with $\mathrm{H}_{0}=70 \mathrm{~km} \mathrm{~s}^{-1} \mathrm{Mpc}^{-1}, \Omega_{\Lambda}=0.7$, and $\Omega_{\mathrm{M}}=0.3$. This gives a scale of $180 \mathrm{pc} /{ }^{\prime \prime}$ at the redshift of NGC 3100 (see Table 1). All of the velocities in this paper are given in the optical convention and Kinematic Local Standard of Rest (LSRK) frame. 


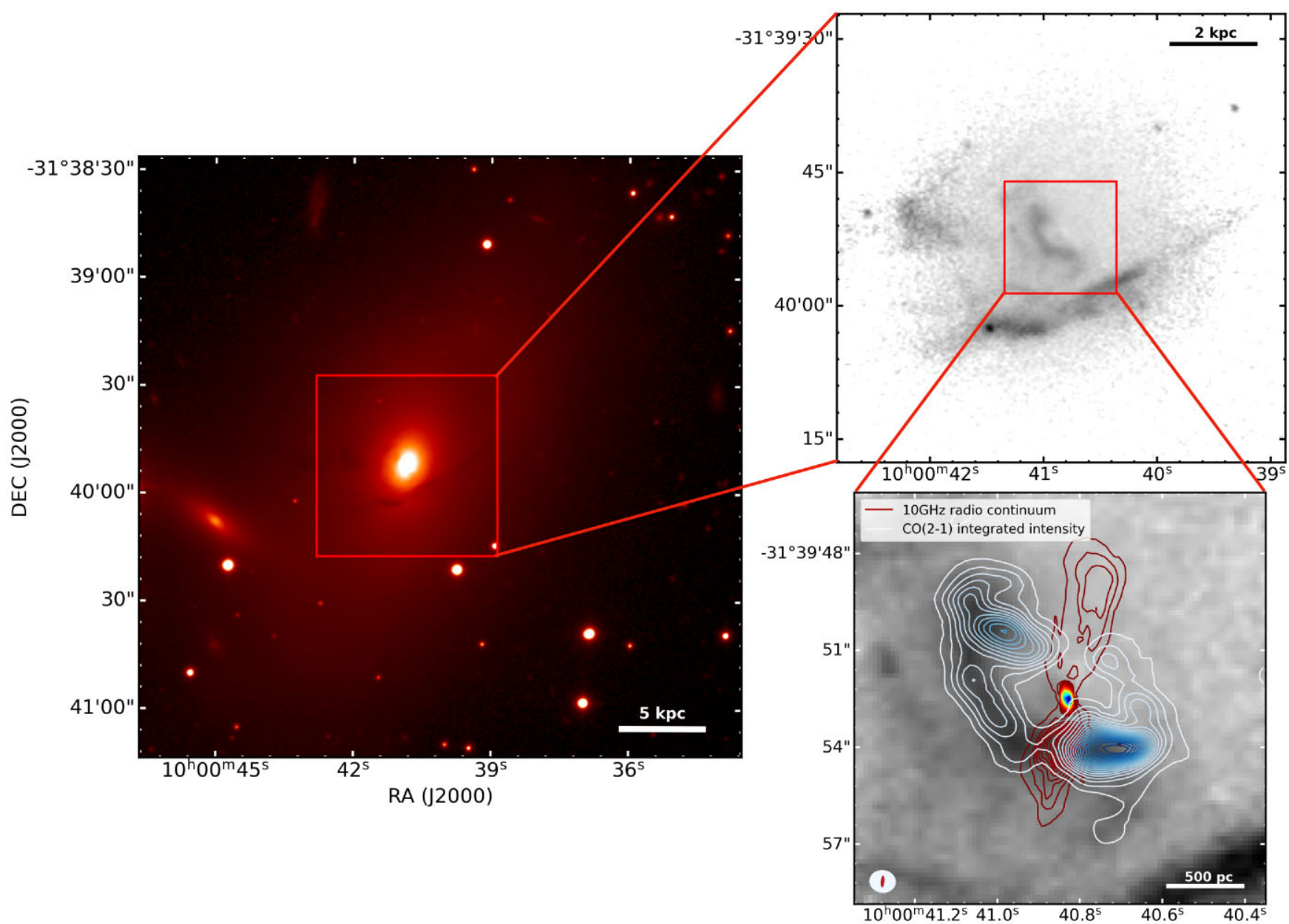

Figure 1. Left-hand panel: Optical image of NGC 3100 from the Carnegie-Irvine Galaxy Survey (CGS; Ho et al. 2011) taken with a blue filter (300-400 nm). The image resolution (i.e. seeing FWHM) is $0.77^{\prime \prime}$. The size of the panel is $167^{\prime \prime} \times 167^{\prime \prime}\left(\approx 30 \times 30 \mathrm{kpc}^{2}\right)$. Right-hand panel, top: B - I colour (dust extinction) map of NGC 3100 in the central $50^{\prime \prime} \times 50^{\prime \prime}\left(\approx 9 \times 9 \mathrm{kpc}^{2}\right.$; see Paper I, for details). Right-hand panel, bottom: As above, but zoomed in the inner $13^{\prime \prime} \times 13^{\prime \prime}$ $\left(\approx 2.3 \times 2.3 \mathrm{kpc}^{2}\right) . \mathrm{CO}(2-1)$ integrated intensity contours from our Cycle 3 ALMA observations (Paper I; Paper II), and radio continuum contours from our JVLA data at $10 \mathrm{GHz}$ (Paper III) are overlaid in light-blue and red contours, respectively. Contours are drawn at 1,3,9 ... times the 3- $\sigma$ rms noise level.

\section{A SOUTHERN RADIO GALAXY SAMPLE}

A full description of our project, including details on the sample selection and all the available observations, can be found in Ruffa et al. (2019a); a brief summary is presented here.

Starting from the southern radio galaxy (RG) sample of Ekers et al. (1989), which lists all the sources in the declination range $-17^{\circ}<$ $\delta<-40^{\circ}$ with radio flux $S_{2.7 \mathrm{GHz}} \geq 0.25 \mathrm{Jy}$ and optical apparent magnitude $m_{\mathrm{v}}<17.0$, we have selected a complete sub-sample of 11 RGs. Based on the available optical spectroscopy (Tadhunter et al. 1993; Smith et al. 2000; Colless et al. 2003; Collobert et al. 2006; Jones et al. 2009; Dopita et al. 2015), all of the 11 sources can be classified as LERGs.

Nine sample members were observed during ALMA Cycle 3 in the ${ }^{12} \mathrm{CO}(2-1) \operatorname{line}^{2}$ and $230-\mathrm{GHz}$ continuum (Ruffa et al. 2019a, hereafter Paper I). These observations show that rotating (sub-)kpc molecular gas discs are very common in LERGs. The 3D modelling of these discs (Ruffa et al. 2019b, hereafter Paper II) demonstrates that although the bulk of the gas appears to be in ordered

${ }^{2}$ Hereafter, we omit the carbon mass number and refer to the ${ }^{12} \mathrm{CO}$ isotopologue as simply CO. rotation, low-amplitude perturbations and/or non-circular motions are ubiquitous and strongly suggestive of the presence of radio jet$\mathrm{CO}$ disc interactions in at least two cases. The comparison between the ALMA CO(2-1) data and newly acquired, high-resolution Karl G. Jansky Very Large Array (JVLA) radio continuum observations at $10 \mathrm{GHz}$ (Ruffa et al. 2020, hereafter Paper III) adds further evidence for the presence of such an interaction in at least one of the two candidates, NGC 3100.

\subsection{A case study: NGC 3100}

NGC 3100 is optically classified as a late S0 galaxy, characterized by a patchy dust distribution and a bright nuclear component (Sandage \& Brucato 1979; Laurikainen et al. 2006; Fig. 1, left-hand panel). It resides in a poor group and forms a pair with the barred spiral galaxy NGC 3095, located at a projected linear distance of $\approx 120 \mathrm{kpc}$ (De Vaucouleurs 1976). NGC 3100 is the host galaxy of the radio source PKS 0958-314 (e.g. Ekers et al. 1989; Paper I; Paper III). A summary of some general target properties is provided in Table 1.

Among the radio galaxies that we imaged in $\mathrm{CO}(2-1)$, NGC 3100 stands out: we detect a well-resolved ring-like central CO structure extending $\approx 1.6 \times 0.5 \mathrm{kpc}^{2}$, observed to be nicely co-spatial with 
Table 2. Main properties of the ALMA observations presented in this paper.

\begin{tabular}{|c|c|c|c|c|c|c|c|c|c|c|c|}
\hline $\begin{array}{l}\text { ALMA } \\
\text { band }\end{array}$ & $\begin{array}{l}\text { Observation } \\
\text { date }\end{array}$ & $\begin{array}{l}\text { Targeted } \\
\text { line }\end{array}$ & $\begin{array}{c}v_{\text {rest }} \\
(\mathrm{GHz})\end{array}$ & $\begin{array}{c}v_{\text {sky }} \\
(\mathrm{GHz})\end{array}$ & $\begin{array}{l}\text { Time } \\
(\mathrm{min})\end{array}$ & $\begin{array}{c}\text { MRS } \\
(\mathrm{kpc})(\operatorname{arcsec})\end{array}$ & $\begin{array}{c}\text { FoV } \\
(\mathrm{kpc})(\operatorname{arcsec})\end{array}$ & $\theta_{\text {maj }}$ & $\theta_{\min }$ & $\begin{array}{l}\mathrm{PA}_{\text {beam }} \\
(\mathrm{deg})\end{array}$ & $\begin{array}{l}\text { Scale } \\
\text { (pc) }\end{array}$ \\
\hline (1) & (2) & (3) & (4) & (5) & (6) & (7) & (8) & (9) & (10) & (11) & (12) \\
\hline Band 3 & $2019-04-23 / 24$ & ${ }^{12} \mathrm{CO}(1-0)$ & 115.2712 & 114.2657 & 62 & $3.7(21)$ & $8.9(50)$ & 1.4 & 1.0 & -85 & 250 \\
\hline Band 4 & $2018-12-14 / 15$ & $\begin{array}{c}\mathrm{HNCO}(6-5) \\
\mathrm{SiO}(3-2)\end{array}$ & $\begin{array}{l}131.8857 \\
130.2686\end{array}$ & $\begin{array}{l}130.7352 \\
129.1322\end{array}$ & 188 & $3.4(19)$ & $7.6(43)$ & 1.1 & 0.9 & $\begin{array}{l}-81 \\
-84\end{array}$ & 195 \\
\hline Band $6^{a}$ & 2016-03-22 & ${ }^{12} \mathrm{CO}(2-1)$ & 230.5380 & 228.6299 & 28 & $1.9(11)$ & $4.4(25)$ & 0.9 & 0.7 & -87 & 160 \\
\hline
\end{tabular}

Notes. Columns: (1) ALMA frequency band. (2) Date of the observation. (3) Targeted molecular transition. (4) Rest-frame central line frequency. (5) Redshifted (sky) central frequency of the line estimated from the redshift listed in row (4) of Table 1. (6) Total integration time-on-source. (7) Maximum recoverable scale (MRS) in kiloparsec, and corresponding scale in arcseconds within parentheses. (8) Field of view (FOV; i.e. primary beam FWHM) in kiloparsec, and corresponding scale in arcseconds within parentheses. (9) and (10) Major and minor axis FWHM of the synthesized beam. (11) Position angle of the synthesized beam. (12) Physical scale corresponding to the major axis FWHM of the synthesized beam.

${ }^{a}$ ALMA observation presented in Paper I and reported here for completeness. See Section 3 for details.

dust (Fig. 1, upper- and bottom right-hand panels). Other structures with patchy morphologies are observed up to $\approx 2 \mathrm{kpc}$ from the central ring (Paper I). The 3D kinematic modelling of the main CO distribution (Paper II) demonstrates that although the bulk of the gas is regularly rotating, there are evident distortions in the velocity field that are best described by the combination of both warps and non-circular motions (with peak velocities around $\pm 40 \mathrm{~km} \mathrm{~s}^{-1}$, corresponding to $\pm 52 \mathrm{~km} \mathrm{~s}^{-1}$ deprojected). ${ }^{3}$ The $10-\mathrm{GHz}$ JVLA observations provide the highest resolution radio continuum map currently available for NGC 3100 (Fig. 1, bottom right-hand panel). As extensively discussed in Paper III, these data show no evidence for highly collimated jet structures on scales larger than the unresolved core, so the inner jets must have disrupted and decollimated close to the region where they were launched. This indicates rapid jet deceleration, which is also consistent with the wide opening-angle radio lobes observed on larger scales in our 10-GHz map. Such lobes are rather asymmetric, both in surface brightness and morphology. The northern lobe, in particular, bends at a position that (at least in projection) seems coincident with a distortion in the $\mathrm{CO}(2-1)$ disc (see Fig. 1, bottom right-hand panel). The total linear extent of the radio source is $\approx 2 \mathrm{kpc}$ and there is no evidence from the available multifrequency radio data for emission on larger scales. This suggests that the radio source in NGC 3100 is likely in an early phase of its evolution (Paper III). More in general, all of the observational properties described above strongly suggest the presence of a (sub-)kpc scale jet-ISM interaction in this object.

\section{ALMA OBSERVATIONS AND DATA REDUCTION}

The observations presented in this paper were carried out during ALMA Cycle 6 between 2018 December and 2019 April (PI: I. Ruffa). Their main properties are summarized in Table 2.

NGC 3100 was observed in three different ALMA Bands: in Band 3, we targeted the $\mathrm{CO}(1-0)$ transition; in Band 4, the $\operatorname{HNCO}(6-5)$ and $\mathrm{SiO}(3-2)$; and in $\mathrm{Band} 7$, the $\mathrm{CO}(3-2)$ and $\mathrm{HCO}^{+}$(4-3) lines. We adopted the same spectral configuration for all of the observations: a total of four spectral windows (SPWs); one (or two, depending on the number of lines targeted in the observing block) centred at the

\footnotetext{
${ }^{3}$ Observed velocities are projected along the line of sight. Intrinsic values can be estimated by correcting for the inclination of the gas distribution, i.e. $v_{\text {deproj }}=v_{\text {obs }} / \sin i$.
}

line(s) redshifted frequency $\left(v_{\text {sky }}\right)$ and divided into $19201.129 \mathrm{MHz}$ wide channels, the other three (or two) used to map the continuum emission and divided into 240 7.813-MHz-wide channels. Between 41 and 48 12-m antennae were used; the maximum baseline length was $783 \mathrm{~m}$ for Band 3 and 4 observations, $360 \mathrm{~m}$ for Band 7 . A standard calibration strategy was adopted: for each session, a single bright quasar was used as both flux and bandpass calibrator, a second one as phase calibrator. Specifically, we used J1037 - 2934 and $\mathrm{J} 1024$ - 3234 for Band 3, J1107 - 4449 and J1037 - 2934 for Band 4, and $\mathrm{J} 1058+0133$ and $\mathrm{J} 1037$ - 2934 for Band 7, respectively.

As already mentioned above, these newly acquired data complement previous Band 6 observations of NGC 3100 in the $\mathrm{CO}(2-1)$ transition, which were obtained in 2016 March as part of ALMA Cycle 3 (PI: I. Prandoni). Full details on these observations can be found in Paper I; a summary is provided in Table 2 for completeness.

We manually calibrated all data using the Common Astronomy Software Application (CASA; McMullin et al. 2007) package, version 5.4.1 (4.7.2 for Band 6 data), reducing each data set separately with customized PYTHON data reduction scripts.

\subsection{Continuum imaging}

The continuum SPWs and the line-free channels of the line SPW(s) of each observing run were used to produce the continuum maps, using the tclean task in multifrequency synthesis mode (MFS; Rau \& Cornwell 2011) with no spectral dependence. All the continuum maps were made using natural weighting in order to maximize the sensitivity, with the goal of imaging extended emission from the radio source. Since continuum emission from the core is detected at high signal-to-noise ratios $(\mathrm{S} / \mathrm{N})$ at all frequencies, multiple cycles of phase-only self-calibration and one cycle of amplitude and phase self-calibration were performed at all frequencies. This allowed us to obtain root-mean square (rms) noise levels ranging from 9 to $50 \mu \mathrm{Jy}$ beam $^{-1}$ for synthesized beams of $0.6-0.9$ arcsec full width at half-maximum (FWHM). Table 3 summarizes the main properties of the millimetre continuum maps, which are shown in Fig. 2. For completeness, we include the 230-GHz continuum map and its properties (originally presented in Paper I) in Fig. 2 and Table 3, respectively. All the continuum maps are primary-beam corrected.

\subsection{Line imaging}

After applying the continuum self-calibration, the $\mathrm{CO}$ line emission was isolated in the $u v$ plane using the CASA task uvcontsub. This 
Table 3. Properties of the continuum images.

\begin{tabular}{lccc}
\hline $\begin{array}{l}\text { ALMA } \\
\text { Band }\end{array}$ & $\begin{array}{l}\text { Continuum } \\
\text { component }\end{array}$ & rms & $S_{v}$ \\
$(1)$ & $(2)$ & $\begin{array}{c}\left(\mu \mathrm{Jy} \mathrm{beam}^{-1}\right) \\
(3)\end{array}$ & $\begin{array}{c}(\mathrm{mJy}) \\
(4)\end{array}$ \\
\hline Band 3 & Core & 15 & $37.0 \pm 3.7$ \\
& N jet & & $5.9 \pm 0.6$ \\
& S jet & & $10 \pm 1.0$ \\
Band 4 & Core & 9 & $28 \pm 2.8$ \\
& N jet & & $4.5 \pm 0.4$ \\
& S jet & & $7.7 \pm 0.8$ \\
Band 6 ${ }^{a}$ & Core & 20 & $43.0 \pm 4.3$ \\
& N jet & & $2.2 \pm 0.2$ \\
& S jet & & $19 \pm 0.4$ \\
Band 7 & Core & 50 & - \\
& N jet & & $3.4 \pm 0.3$ \\
& S jet & & \\
\hline
\end{tabular}

Notes. Columns: (1) ALMA frequency band. (2) Component identified in the mm continuum maps. (3) rms noise level measured from emission-free regions of the cleaned continuum map. (4) Integrated continuum flux density of the components listed in column (2) and corresponding uncertainties. These latter are estimated as $\sqrt{\mathrm{rms}^{2}+\left(0.1 \times S_{v}\right)^{2}}$, where the second term dominates in all cases.

${ }^{a}$ Continuum properties of the Band 6 continuum map from Paper I. See Section 3 for details.

${ }^{b}$ Undetected component.

forms a continuum model from linear fits in frequency to line-free channels and then subtracts this model from the visibilities.

We created the data cubes of the $\mathrm{CO}$ transitions using the tclean task with natural weighting and channel widths of $10 \mathrm{~km} \mathrm{~s}^{-1}$. The channel velocities were computed in the source frame with zeropoints corresponding to the redshifted frequency of the corresponding line (i.e. $v_{\text {sky }}$; see Table 2 ). The continuum-subtracted dirty cubes were cleaned in regions of line emission (identified interactively) to a threshold equal to 1.5 times the rms noise level (determined from line-free channels) and then primary-beam corrected. $\mathrm{CO}(1-0)$ and $\mathrm{CO}(3-2)$ emission is clearly detected, with peak $\mathrm{S} / \mathrm{N}$ of 23 and 83, respectively, and an rms noise (determined from line-free channels) of $0.6 \mathrm{mJy}_{\text {beam }}^{-1}$ in both cases.

$\mathrm{SiO}(3-2), \mathrm{HNCO}(6-5)$, and $\mathrm{HCO}^{+}(4-3)$ emission (and consequently line-free channels) could not be identified in the SPWs centred on the corresponding redshifted line frequencies; the continuum was thus modelled using the continuum SPWs only. Continuumsubtracted data cubes of these transitions were created with a conservative channel width of $105 \mathrm{~km} \mathrm{~s}^{-1}$ and natural weighting. The resulting dirty cubes were then cleaned down to 1.5 times the expected rms noise, yielding cleaned channel maps with 1- $\sigma$ rms noises ranging from 0.05 to $0.2 \mathrm{mJy}$ beam $^{-1}$. The $\mathrm{SiO}(3-2)$ and $\mathrm{HNCO}(6-5)$ lines are undetected, while $\mathrm{HCO}^{+}(4-3)$ emission is tentatively detected at $\approx 5 \sigma$.

Table 4 summarizes the properties of the imaged data cubes [including $\mathrm{CO}(2-1)$ from Paper I]; for non-detections, 3- $\sigma$ upper limits are tabulated.

\section{IMAGE CUBE ANALYSIS AND RESULTS}

\subsection{Moment maps}

Integrated intensity (moment 0 ), mean line-of-sight velocity (moment 1 ), and velocity dispersion (moment 2 ) maps of the CO lines were created from the continuum-subtracted data cubes using the masked moment technique as described by Dame (2011, see also Bosma 1981a,b; van der Kruit \& Shostak 1982; Rupen 1999). In this technique, a copy of the cleaned data cube is first Gaussian-smoothed spatially (with an FWHM equal to that of the synthesized beam) and then Hanning-smoothed in velocity. A 3D mask is then defined by selecting all the pixels above a fixed flux-density threshold. This threshold is chosen so as to recover as much flux as possible while minimizing the noise (higher thresholds usually need to be set for noisier maps; see also Paper I). Given the high significance of our CO detections (see Table 4), a threshold of $1.2 \sigma$ (where $\sigma$ is the rms noise level measured in the un-smoothed data cube) has been used in all the cases. The moment maps were then produced from the un-smoothed cubes using the masked regions only (e.g. Davis et al. 2017). The resulting moment maps of the CO transitions [including those of the $\mathrm{CO}(2-1)$ from Paper I] are shown in Fig. 3.

It is clear from the left-hand panels of Fig. 3 that for all the $\mathrm{CO}$ transitions, the bulk of the emission arises from two bright peaks centred approximately at RA offsets of $1^{\prime \prime}(\approx 190 \mathrm{pc})$ to the north-east (NE) and south-west (SW) of the core. These features are surrounded by fainter emission extending up to $11^{\prime \prime}(\approx 2 \mathrm{kpc})$ along the major axis of the $\mathrm{CO}(1-0)$ distribution, a bit less $(\approx 1.6 \mathrm{kpc})$ in the higher$J$ transitions. In general, substantial differences can be observed between the three lines in terms of the relative brightness of the inner and outer regions: higher- $J$ transitions are brighter towards the core, whereas lower- $J$ transitions are brighter towards the outskirts. As a result, also the gas morphology changes: a gap is observed at the centre of the $\mathrm{CO}(1-0)$ distribution, whereas the $\mathrm{CO}(2-1)$ and $\mathrm{CO}(3-$ 2) lines show a ring-like and full-disc morphology, respectively. Additional detached $\mathrm{CO}$ structures with lower surface brightness are visible at $\approx 1 \mathrm{kpc}$ west and $\approx 2 \mathrm{kpc}$ east from the outer edges of the central CO distribution (Fig. 3). The latter, in particular, is clearly more prominent in the 1-0 than in the 2-1 transition, while it is not observed in $\mathrm{CO}(3-2)$. This seems to confirm the general trend for lower- $J$ lines to be brighter farther away from the centre, although we note that at least some of the $\mathrm{CO}(2-1)$ emission of the eastern patch may be lost due to significant primary beam attenuation in that region at $235 \mathrm{GHz}$ [Field of view $(\mathrm{FoV})=25^{\prime \prime}$; see Table 2]. Primary beam attenuation/limitation may also explain the non-detection of any emission from the eastern patch in Band 7 observations $\left(\mathrm{FoV}=17^{\prime \prime}\right.$; see Table 2$)$. The relative spatial distribution of the CO lines suggests higher gas excitation in the central regions. This is further analysed in Section 4.4 and discussed in Section 5.2.

The velocity pattern of the three CO transitions (Fig. 3, middle panels) shows that the gas is rotating but with evident kinematic distortions (i.e. s-shaped iso-velocity contours). Such disturbances usually trace the presence of unrelaxed substructures in the gas disc, possibly caused by either warps or non-circular motions, or a combination of both. Accurate 3D kinematic modelling can help differentiating between the two. This is illustrated in Section 4.5.

The line-of-sight gas velocity dispersion ( $\sigma_{\text {gas }}$; Fig. 3, right-hand panels) varies significantly across the gas sky distributions. In all the transitions, the gas is observed as being dynamically cold at the outskirts of the main $\mathrm{CO}$ structure and at larger scales, with $\sigma_{\text {gas }} \approx 10-20 \mathrm{~km} \mathrm{~s}^{-1}$. These values are in agreement with the typical ranges measured in nearby cold gas clouds (e.g. van de Voort et al. 2018). The gas velocity dispersion then progressively increases towards the central gas regions but differently from lower- to higher- $J$ transitions. In both the 1-0 and 2-1 lines, the highest values $\left(\geq 50 \mathrm{~km} \mathrm{~s}^{-1}\right)$ are almost exclusively concentrated in areas spatially coincident with the location of the two line peaks. In the $\mathrm{CO}(3-2)$, we instead observe large line broadening 
(a) Band 3

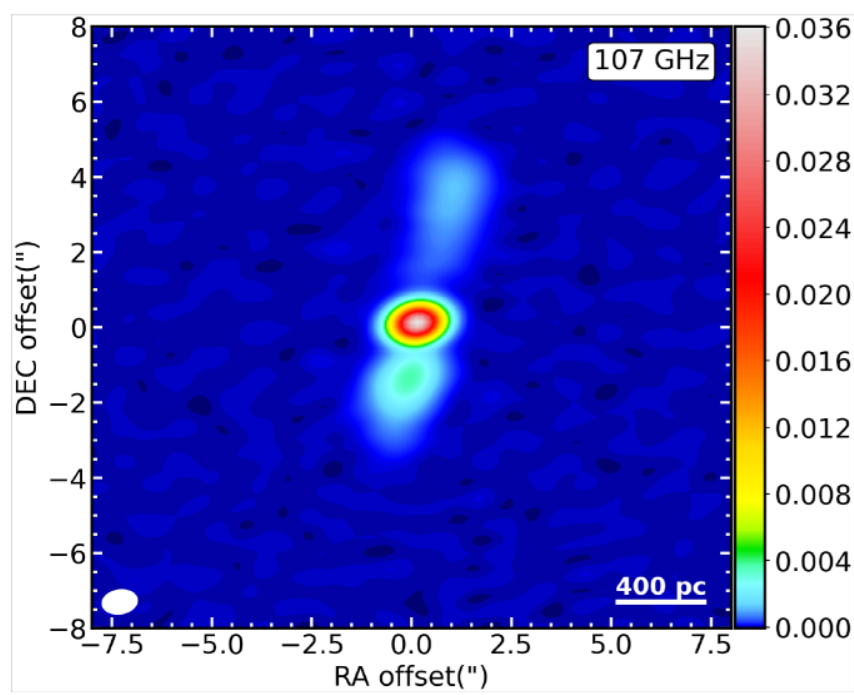

(c) Band 6

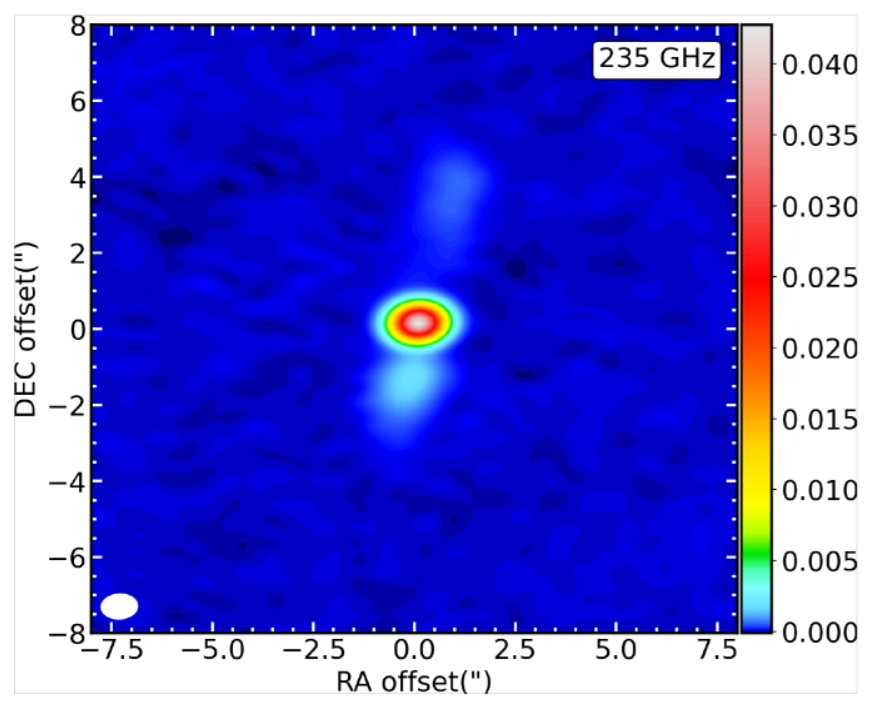

(b) Band 4

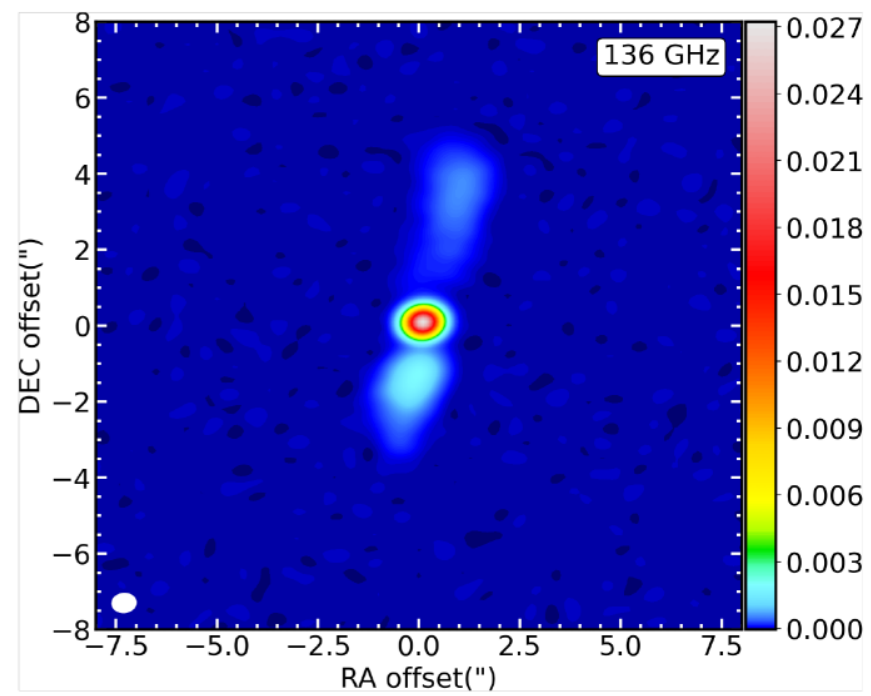

(d) Band 7

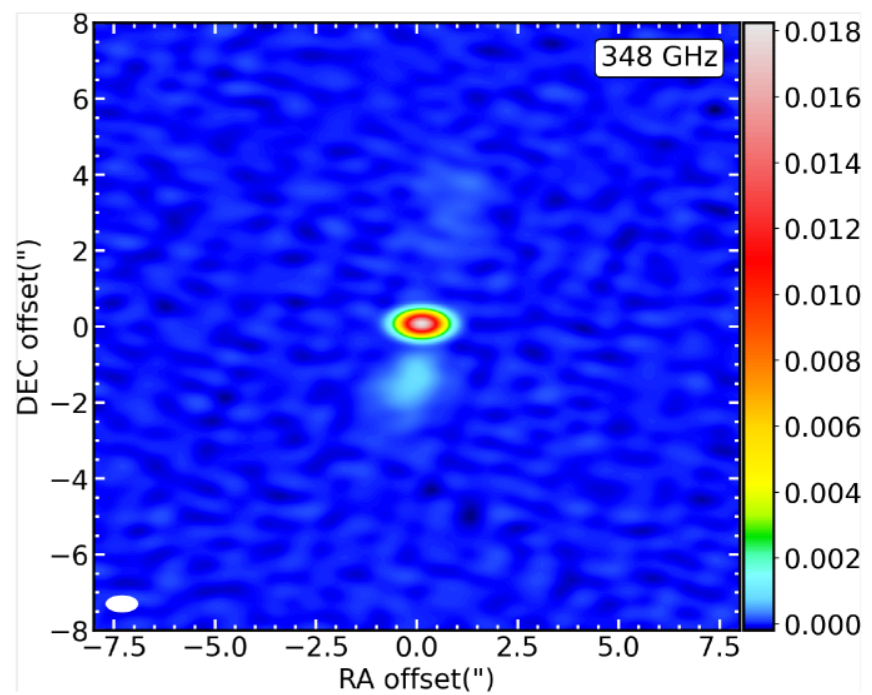

Figure 2. Naturally weighted (sub-)mm continuum maps of NGC 3100 in ALMA Band 3 (panel a), 4 (panel b), 6 (panel c), and 7 (panel d). The Band 6 image is from Paper I. The reference frequency of each map is indicated in its top right corner. The bar to the right of each map shows the colour scale in Jy beam ${ }^{-1}$. Coordinates are given as relative to the image phase centre; East is to the left and North to the top. The synthesized beam is shown in white in the bottom left corner of each panel; a scale bar is also shown in the bottom right corner of panel a. The properties of the continuum maps are summarized in Table 3.

Table 4. Properties of the imaged data cubes.

\begin{tabular}{lcccc}
\hline Transition & $\begin{array}{c}\text { rms } \\
\left(\mathrm{mJy} \mathrm{beam}^{-1}\right) \\
(2)\end{array}$ & $\begin{array}{c}\text { Peak flux } \\
\left(\mathrm{mJy} \mathrm{beam}^{-1}\right)\end{array}$ & $\begin{array}{c}\mathrm{S} / \mathrm{N} \\
(3)\end{array}$ & $\begin{array}{c}\Delta v_{\text {chan }} \\
\left(\mathrm{km} \mathrm{s}^{-1}\right) \\
(5)\end{array}$ \\
\hline${ }^{12} \mathrm{CO}(1-0)$ & 0.6 & 13.8 & 23 & 10 \\
$\mathrm{HNCO}(6-5)$ & 0.05 & $<0.15$ & - & 105 \\
$\mathrm{SiO}(3-2)$ & 0.05 & $<0.15$ & - & 105 \\
${ }^{12} \mathrm{CO}(2-1)^{a}$ & 0.6 & 28.3 & 45 & 10 \\
$\mathrm{HCO}^{+}(4-3)$ & 0.2 & 1.0 & 5 & 105 \\
${ }^{12} \mathrm{CO}(3-2)$ & 0.6 & 50.0 & 83 & 10 \\
\hline
\end{tabular}

Notes. Columns: (1) Line transition. (2) rms noise measured from line-free channels with the widths listed in column (5). (3) Peak flux of the line emission. (4) Peak signal-to-noise ratio of the detection. (5) Channel width of the data cube $\left(\mathrm{km} \mathrm{s}^{-1}\right.$ in the source frame).

${ }^{a}$ Properties of the $\mathrm{CO}(2-1)$ data cube presented in Paper I. See Section 3 for details. $\left(\sigma_{\text {gas }} \geq 40 \mathrm{~km} \mathrm{~s}^{-1}\right)$ throughout the central regions of the main gas structure. Such large values would imply that an ongoing perturbation (such as deviations from purely circular motions) is affecting the dynamical state of the gas, inducing turbulence within the gas clouds. Caution is needed, however, as observed line-of-sight velocity dispersions can be significantly overestimated due to observational effects. Among these, beam smearing (i.e. contamination from partially resolved velocity gradients within the host galaxy) usually dominates in regions of large velocity gradients, such as the inner regions of galaxies. Full kinematic modelling is the only way to derive reliable estimates of the intrinsic gas velocity dispersion (see Section 4.5).

A masked moment 0 map of the detected $\mathrm{HCO}^{+}(4-3)$ emission is shown in Fig. 4, with $\mathrm{CO}(3-2)$ contours overlaid. The significance of the detection is not sufficient to produce reliable higher moment maps (line emission can be identified only in four channels of the data 
(a) $\operatorname{CO}(1-0)$ moments
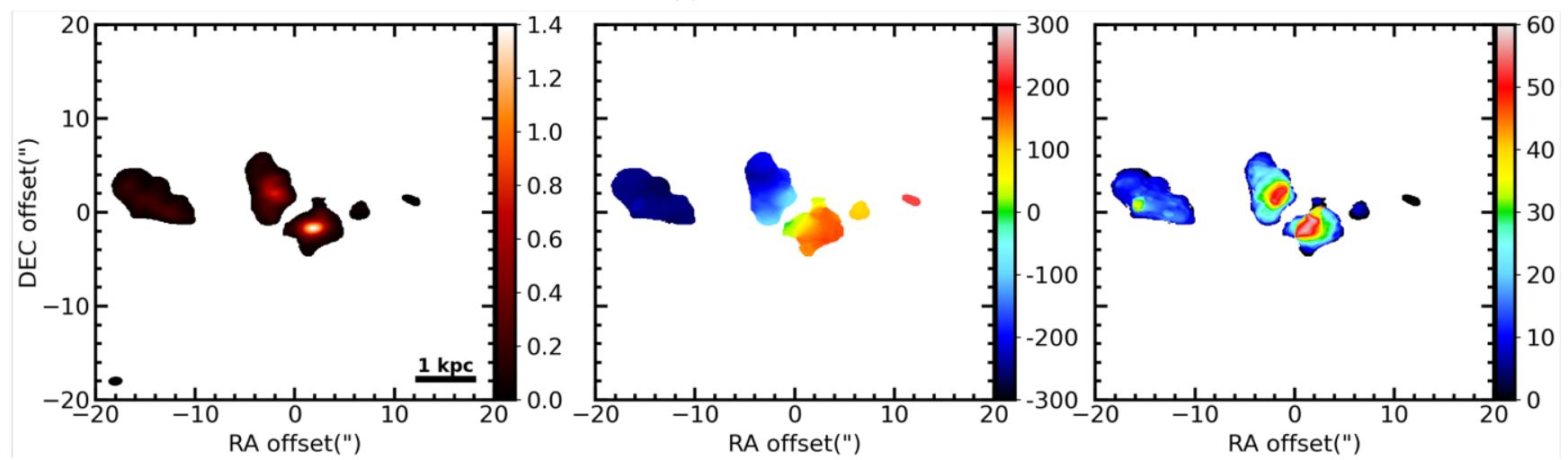

(b) $\operatorname{CO}(2-1)$ moments
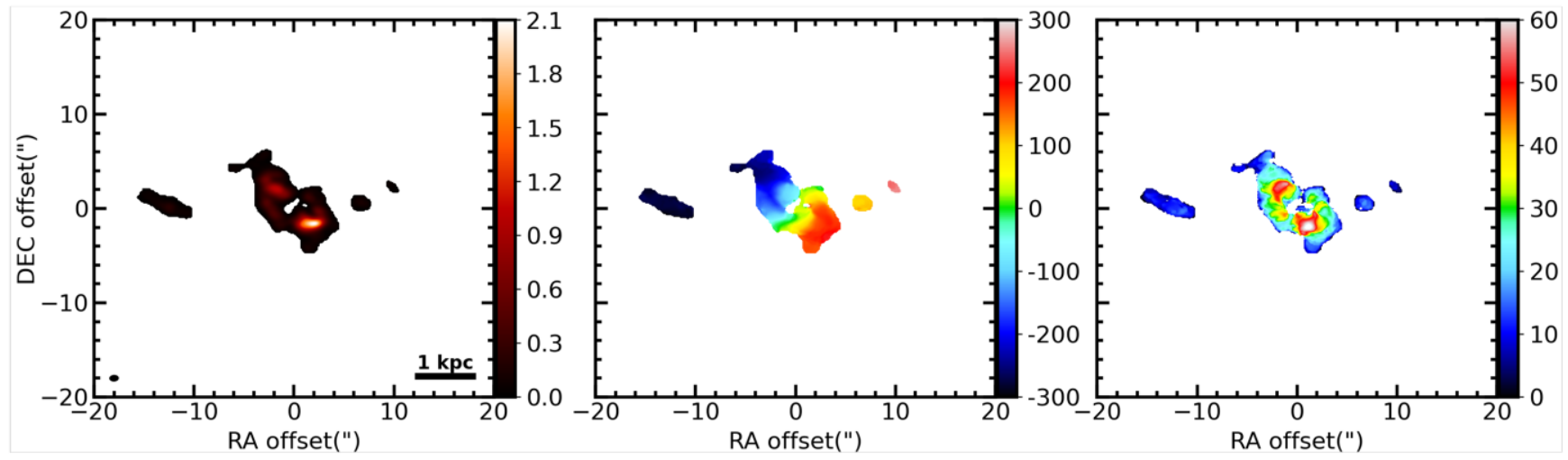

(c) $\operatorname{CO}(3-2)$ moments
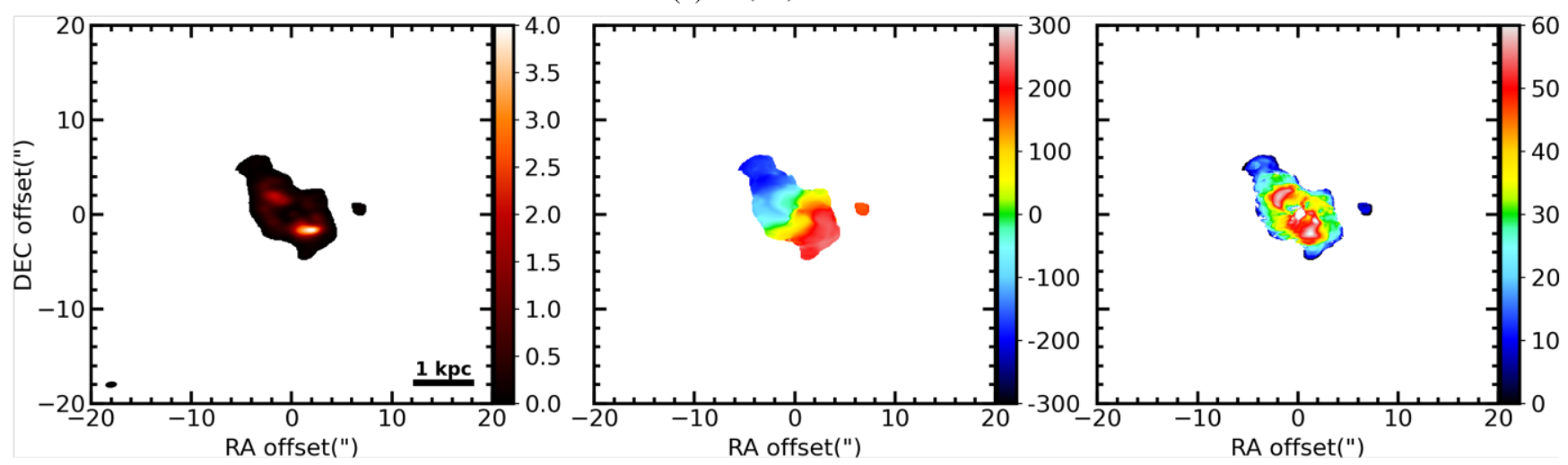

Figure 3. NGC 3100 moment 0 (integrated intensity; left-hand panels), moment 1 (intensity-weighted mean line-of-sight velocity; middle panels) and moment 2 (intensity-weighted line-of-sight velocity dispersion; right-hand panels) maps of the $\mathrm{CO}(1-0), \mathrm{CO}(2-1)$, and $\mathrm{CO}(3-2)$ transitions (top, middle, and bottom rows, respectively). The maps were created with the masked moment technique described in Section 4.1 using data cubes with a channel width of $10 \mathrm{~km} \mathrm{~s}^{-1}$ (see Table 4). The synthesized beam and a scale bar are shown in the bottom-left and bottom-right corner, respectively, of each moment 0 map. The bar to the right of each map shows the colour scales (in Jy beam ${ }^{-1} \mathrm{~km} \mathrm{~s}^{-1}$ and $\mathrm{km} \mathrm{s}^{-1}$ for moment 0 and moment $1 / 2$ maps, respectively). Coordinates are with respect to the image phase centre; East is to the left and North to the top. Velocities are measured in the source frame and the zero-point corresponds to the intensity-weighted centroid of the $\mathrm{CO}$ emission.

cube, with a peak $\mathrm{S} / \mathrm{N}=5$; see Table 4 and Section 4.2). The faint $\mathrm{HCO}^{+}(4-3)$ detection is restricted to a single unresolved blob-like structure at $2^{\prime \prime}(\approx 350 \mathrm{pc})$ west of the core, not coincident with the position of any of the $\mathrm{CO}$ peaks. Interestingly, $\mathrm{HCO}^{+}(4-3)$ emission is detected only at blueshifted velocities (see Section 4.2), while it is located in a small region of the receding (i.e. redshifted) $\mathrm{CO}$ side. This suggests that the two molecular species have opposite velocity gradients (i.e. they are kinematically decoupled) in that region. This is discussed in Section 5.2.2.

\subsection{Line widths and profiles}

The spectral profiles of the central $\mathrm{CO}(1-0)$ and $\mathrm{CO}(3-2)$ structures were extracted from the cleaned data cubes within boxes covering the same $10^{\prime \prime} \times 11^{\prime \prime}$ central area of each cube. These spectra are shown in Fig. 5(a), together with the $\mathrm{CO}(2-1)$ spectrum from Paper I (extracted within the same region). The spectral profile of the detected $\mathrm{HCO}^{+}(4-$ 3 ) emission, extracted from the cleaned data cube within a $1.5^{\prime \prime} \times$ $1.5^{\prime \prime}$ box centred on the emission peak, is shown in Fig. 5(b). Line 


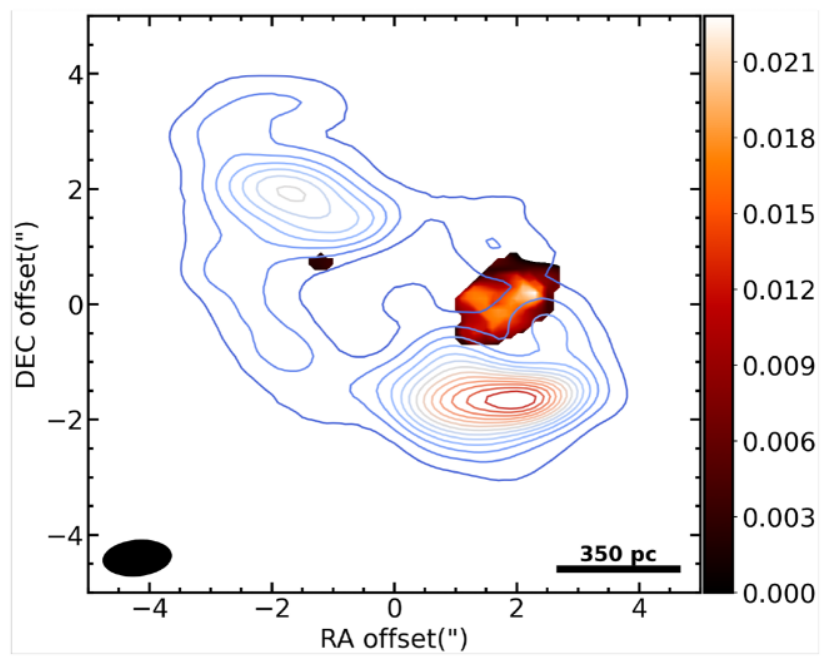

Figure 4. NGC 3100 moment 0 (integrated intensity) map of the $\mathrm{HCO}^{+}(4-3)$ transition, with $\mathrm{CO}(3-2)$ contours overlaid in a cool-to-warm colour scale. The map was created with the masked moment technique described in Section 4.1 using a data cube with a channel width of $105 \mathrm{~km} \mathrm{~s}^{-1}$ (see Table 4). The synthesized beam is shown in the bottom-left corner, and a scale bar in the bottom-right corner. The colour bar to the right shows the flux density scale in Jy beam ${ }^{-1} \mathrm{~km} \mathrm{~s}^{-1}$. Coordinates are relative to the image phase centre; East is to the left and North to the top.

widths were measured as both full width at zero-intensity (FWZI) and FWHM. The former was defined as the full velocity range covered by channels (identified interactively in the channel maps) with line intensities $\geq 3 \sigma$. These channels are highlighted by the shaded regions in Fig. 5. The integrated flux densities listed are calculated over this range. For all the $\mathrm{CO}$ transitions, these values include the flux densities of the larger-scale eastern/western CO structures calculated over the FWZI range of their respective spectral profiles (not shown here).

Different methods were adopted to estimate the line FWHM, depending on the shape of the observed line profile. For the $\mathrm{CO}$ lines, showing the classic double-horned shape of a rotating disc, each FWHM was defined directly from the integrated spectrum as the velocity difference between the two channels with intensities exceeding half of the line peak most distant from the line centre. For the $\mathrm{HCO}^{+}(4-3)$ line, that is centrally peaked, we estimated the FWHM by performing a Gaussian fit on the integrated spectrum (see Fig. 5b).

For the undetected $\mathrm{SiO}(3-2)$ and $\mathrm{HNCO}(6-5)$ shock tracers, $3 \sigma$ upper limits on the velocity-integrated flux densities were calculated from the relation (e.g. Koay et al. 2016):

$S \Delta \mathrm{v}<3 \sigma \Delta \mathrm{v}_{\mathrm{FWHM}} \sqrt{\frac{\Delta \mathrm{v}}{\Delta \mathrm{v}_{\mathrm{FWHM}}}}$,

where $\Delta \mathrm{v}$ is the channel width of the data cube in which the rms noise $(\sigma)$ is measured (105 $\mathrm{km} \mathrm{s}^{-1}$ in these cases; Table 4), and $\Delta v_{\text {FWHM }}$ is the expected line FWHM. We assumed the same FWHM of the $\mathrm{CO}(2-1)$ line (see Table 5). The factor $\sqrt{\Delta \mathrm{v} / \Delta \mathrm{v}_{\mathrm{FWHM}}}$ accounts for the expected decrease in noise level with increasing bandwidth (Wrobel \& Walker 1999). We note that equation (1) is valid only if all of the molecular gas is concentrated within the synthesized beam $\left(\approx 190 \times 160 \mathrm{pc}^{2}\right.$ for Band 4 observations; Table 2$)$. If the molecular gas is actually distributed on larger scales, the quoted upper limits would be underestimated.

\section{(a) CO spectra}

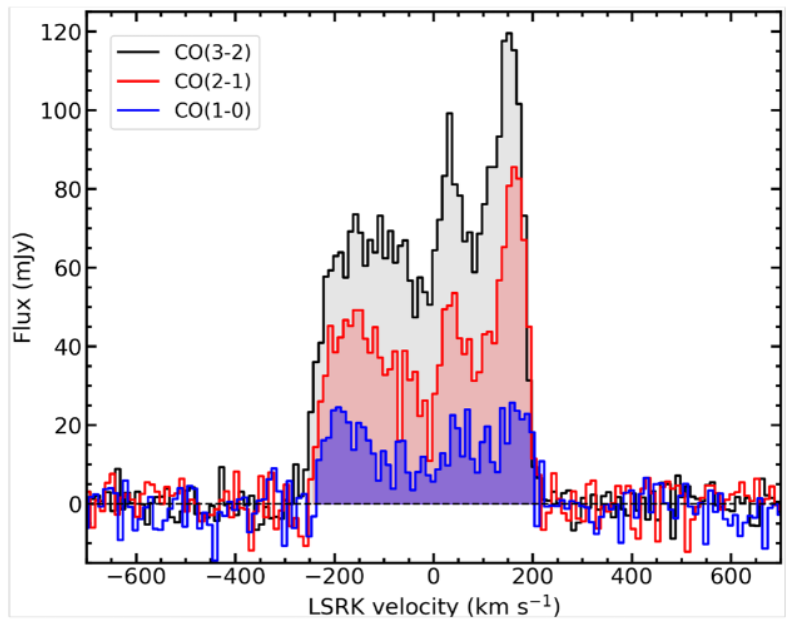

(b) $\mathrm{HCO}^{+}(4-3)$ spectrum

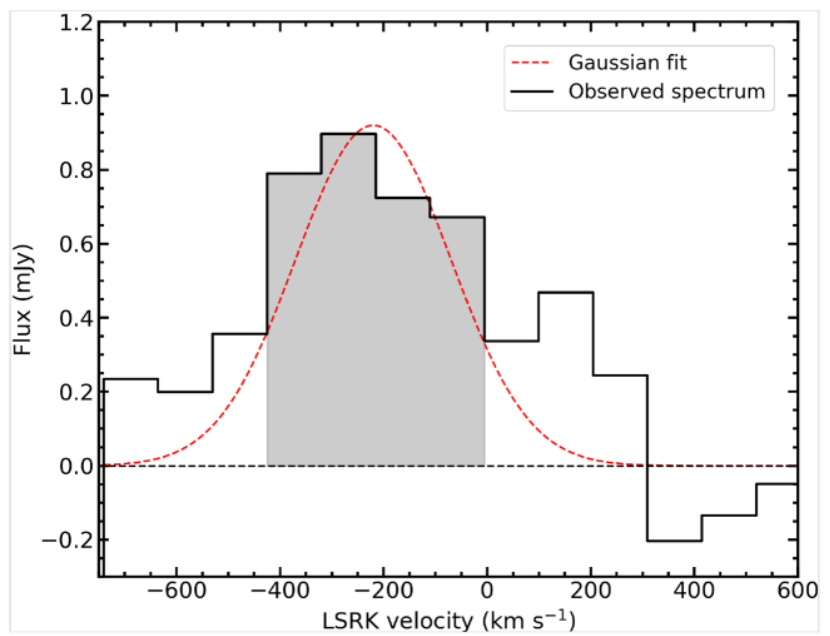

Figure 5. Top panel: $\mathrm{CO}(3-2), \mathrm{CO}(2-1)$, and $\mathrm{CO}(1-0)$ spectral profiles (colours as labelled in the top left corner) extracted within boxes of $10^{\prime \prime} \times$ $11^{\prime \prime}$, including all the emission of the main CO structure visible in Fig. 3. Bottom panel: $\mathrm{HCO}^{+}(4-3)$ spectrum (black solid line) extracted within a box of $1.5^{\prime \prime} \times 1.5^{\prime \prime}$, including all the detected $\mathrm{HCO}^{+}$emission. The bestfitting Gaussian profile is overlaid as a red dashed line. In both panels, the black dashed horizontal line indicates the zero flux level. The shaded regions highlight the spectral channels used to estimate the line FWZI (see Section 4.2 for details).

The measured FWZI, FWHM, and integrated flux densities of the detected lines, along with the upper limits of non-detections, are reported in Table 5 .

\subsection{Molecular gas mass}

An estimate of the molecular gas mass of NGC 3100 was already provided in Paper I, based on our previous $\mathrm{CO}(2-1)$ observations. The successful detection of the $\mathrm{CO}(1-0)$ transition, however, allows us to obtain a more accurate estimate by removing the uncertainties introduced by the assumed $\mathrm{CO}(2-1)$ to $\mathrm{CO}(1-0)$ average flux density ratio (i.e. the ratio of the integrated flux density at each transition).

We adopted the following relation to estimate the total molecular gas mass, including contributions from heavy elements 
Table 5. Main integrated parameters of the observed molecular transitions.

\begin{tabular}{lcccc}
\hline Transition & $\begin{array}{c}\text { Line FWHM } \\
\left(\mathrm{km} \mathrm{s}^{-1}\right) \\
(2)\end{array}$ & $\begin{array}{c}\text { Line FWZI } \\
\left(\mathrm{km} \mathrm{s}^{-1}\right) \\
(3)\end{array}$ & $\begin{array}{c}S \Delta \mathrm{v} \\
\left(\mathrm{Jy} \mathrm{km} \mathrm{s}^{-1}\right)\end{array}$ & $\mathrm{R}_{\mathrm{J} 1}$ \\
\hline${ }^{12} \mathrm{CO}(1-0)$ & 350 & 440 & $9.3 \pm 1.1$ & - \\
$\mathrm{HNCO}(6-5)$ & 340 & - & $<28$ & \\
$\mathrm{SiO}(3-2)$ & 340 & - & $<28$ & \\
${ }^{12} \mathrm{CO}(2-1)$ & 340 & 440 & $20.0 \pm 2.1^{a}$ & $2.14 \pm 0.4$ \\
$\mathrm{HCO}^{+}(4-3)$ & 150 & 420 & $0.32 \pm 0.2$ & - \\
${ }^{12} \mathrm{CO}(3-2)$ & 350 & 460 & $31.3 \pm 3.2$ & $3.35 \pm 0.6$ \\
\hline
\end{tabular}

Notes. Columns: (1) Molecular transition. (2) Line FWHM. For the CO detections, this is defined directly from the spectral profiles as the velocity difference between the two channels with intensities $\geq 50 \%$ of the line peak most distant from the line centre. For the $\mathrm{HCO}^{+}(4-3)$ line, the FWHM is estimated from a Gaussian fit to the integrated spectrum (red dashed line in Fig. 5b). (3) Full velocity range covered by channels (identified interactively in the channel maps) with intensities $\geq 3 \sigma$ (shaded regions in Fig. 5). (4) Flux density integrated over all the channels in the range defined by the FWZI (for $\mathrm{CO}$ transitions, the emission of the detached larger-scale eastern/western $\mathrm{CO}$ structures visible in Fig. 3 is included). For non-detections, velocityintegrated upper limits (in units of Jy beam ${ }^{-1} \mathrm{~km} \mathrm{~s}^{-1}$ ) are estimated assuming that the emission is unresolved and the line FWHM is the same as that of the $\mathrm{CO}(2-1)$ (see Section 4.2). (5) Average ratio of the higher- $J$ CO lines over the 1-0 transition (i.e. ratio of the integrated flux density at each transition). ${ }^{a}$ Due to a more accurate numerical integration, the integrated $\mathrm{CO}(2-1)$ flux density reported here is slightly larger than that tabulated in Paper I.

( $M_{\text {mol }}$; Bolatto et al. 2013):

$$
\begin{aligned}
M_{\mathrm{mol}}= & 1.05 \times 10^{4}\left(\frac{X_{\mathrm{CO}}}{2 \times 10^{20} \frac{\mathrm{cm}^{-2}}{\mathrm{~K} \mathrm{~km} \mathrm{~s}^{-1}}}\right) \\
& \times\left(\frac{1}{1+z}\right)\left(\frac{S_{\mathrm{CO}} \Delta v}{\mathrm{Jy} \mathrm{km} \mathrm{s}^{-1}}\right)\left(\frac{D_{\mathrm{L}}}{\mathrm{Mpc}}\right)^{2},
\end{aligned}
$$

where $S_{\mathrm{CO}} \Delta \mathrm{v}$ is the $\mathrm{CO}(1-0)$ integrated flux density, $z$ the galaxy redshift, $D_{\mathrm{L}}$ the luminosity distance, and $X_{\mathrm{CO}}$ the CO-to- $\mathrm{H}_{2}$ conversion factor. This latter depends on the molecular gas conditions (e.g. excitation, dynamics, geometry, and stability of single molecular clouds) and other ISM properties (e.g. metallicity) and is therefore likely to vary systematically between different galaxy types (see Bolatto et al. 2013). As introduced in Section 4.1 (see also Sections 4.4 and 5.2), the molecular gas in NGC 3100 displays different excitation conditions in the inner areas of the gas distribution. However, this effect is confined in the regions where $\mathrm{CO}(1-0)$ is mostly undetected and can be thus neglected for the purpose of estimating the molecular gas mass from this transition. More in general, little is known about $X_{\mathrm{CO}}$ in nearby ETGs, as most of the studies carried out so far are focused on late-type disc galaxies. In the very few massive nearby ETGs in which accurate measurements have been made, the average conversion factor has been found to be comparable to that estimated in the Milky Way (see e.g. Utomo et al. 2015), which is thus the value commonly used for local radio galaxies (e.g. Tremblay et al. 2016). Therefore, we adopt an average Milky Way CO-to- $\mathrm{H}_{2}$ conversion factor of $X_{\mathrm{CO}}=$ $2 \times 10^{20} \mathrm{~cm}^{-2}\left(\mathrm{~K} \mathrm{~km} \mathrm{~s}^{-1}\right)^{-1}$.

The estimated molecular gas mass is $(1.4 \pm 0.2 \pm 0.3) \times 10^{8} \mathrm{M}_{\odot}$. This is fully consistent with that estimated from $\mathrm{CO}(2-1)$ data $\left(M_{\text {mol }}=(1.2 \pm 0.1 \pm 0.3) \times 10^{8} \mathrm{M}_{\odot}\right.$; Paper I $)$, despite the average flux density ratio assumed for that calculation (i.e. $R_{21}=2.32$; Paper I) was slightly larger than that measured $\left(R_{21}=2.14 \pm 0.4\right.$; Table 5). This overestimation, however, was counterbalanced by a slight underestimation of the $\mathrm{CO}(2-1)$ integrated flux density. A new estimate of the latter, obtained from a more accurate numerical integration, is reported in Table 5. We note that the first error quoted on the molecular gas mass is random and largely dominated by the uncertainty on the integrated flux density, while the second is systematic and accounts for a 10 per cent uncertainty in the assumed distance. An estimate of the systematic uncertainty introduced by our assumption on $X_{\mathrm{CO}}$ is not included in the quoted error budget. This can be quantified by assuming a conservative scatter of \pm 0.4 dex (about a factor of 2.5; see Bolatto et al. 2013) around the adopted Milky Way value, leading the molecular gas mass to vary within the range of $0.5-3.5 \times 10^{8} \mathrm{M}_{\odot}$.

\subsection{Spatially resolved line ratios}

One of the main aims of the present work is to carry out a spatially resolved analysis of the strength of the observed higher- $J$ CO transitions relative to the lowest order transition. To do this, the data cubes used to make ratio maps need to be identical. We thus re-imaged the three $\mathrm{CO}$ transitions making new data cubes with the same number of channels, channel width (i.e. $10 \mathrm{~km} \mathrm{~s}^{-1}$ ), pixel, and image size. We also corrected for the different $u v$ coverages by smoothing to a common resolution (i.e. selecting a common $u v$ range and convolving to a common synthesized beam). This resulted in data cubes with synthesized beams of $1^{\prime \prime} .4 \times 0^{\prime \prime} .9$ (corresponding to $\approx 250 \times 160 \mathrm{pc}^{2}$ ). Masked integrated intensity maps of these smoothed data cubes were produced and then used to make ratio maps using the CASA task immath. The resulting $R_{21} \equiv S_{\mathrm{CO}(2-1)} / S_{\mathrm{CO}(1-0)}$ and $R_{31} \equiv S_{\mathrm{CO}(3-2)} / S_{\mathrm{CO}(1-0)}$ maps $^{4}$ are shown in Fig. 6, with $10-\mathrm{GHz}$ continuum contours overlaid in black. As already clear from the relative spatial distribution of the three CO lines (see Section 4.1), we can identify at least two distinct molecular gas components from Fig. 6: an outer low-excitation component with flux density ratios mostly between $\approx 1$ and $\approx 4$ and an inner high-excitation component with $R_{\mathrm{j} 1} \geq 8$ and $R_{31}$ reaching values $\geq 12$ (Fig. $6 \mathrm{~b}$ ). The former are compatible with the $\mathrm{CO}$ gas being on average in sub-thermal excitation condition (e.g. Cormier et al. 2018); in some cases, similar ratios have been also associated to gas in an optically thick regime (e.g. Dasyra et al. 2016). The large inner ratios instead imply that the gas is likely thermalized (i.e. at densities above $3000 \mathrm{~cm}^{-3}$ ) and optically thin, since they are significantly above the maximum values of $R_{21}=4$ and $R_{31}=9$ for optically thick emission $(\approx 1$ for ratios in brightness temperature; see e.g. Dasyra et al. 2016; Oosterloo et al. 2017). Assuming local thermodynamic equilibrium (LTE) and optically thin conditions, $\mathrm{CO}$ ratios $\gtrsim 12$ imply gas excitation temperatures $T_{\mathrm{ex}} \gtrsim 50 \mathrm{~K}$ (e.g. Oosterloo et al. 2017). Such extreme conditions are very similar to those observed in the regions of jet-ISM interactions in the few (mostly Seyfert-like) objects in which a multiline study like the one presented in this paper has been carried out, such as NGC 1068 (García-Burillo et al. 2014) and IC 5063 (Dasyra et al. 2016; Oosterloo et al. 2017). By

${ }^{4}$ We note that in this work, we use integrated flux density ratios (expressed in $\mathrm{Jy} \mathrm{km} \mathrm{s}^{-1}$ ). Indeed, although naturally thought to be dimensionless, these are different from integrated brightness temperature ratios (expressed in $\mathrm{K} \mathrm{km} \mathrm{s}^{-1}$ ). This is because, based on the Rayleigh-Jeans formula, the conversion between $\mathrm{K}$ and $\mathrm{J}$ is not linear:

$$
\left(\frac{T}{\mathrm{~K}}\right)=1.22 \times 10^{3}\left(\frac{S}{\mathrm{Jy}}\right)\left(\frac{v}{\mathrm{GHz}}\right)^{-2}\left(\frac{\theta_{\mathrm{maj}} \times \theta_{\min }}{\operatorname{arcsec} \times \operatorname{arcsec}}\right)^{-1},
$$

where $T$ is the brightness temperature, $S$ is the flux density, $v$ is the observing frequency, and $\theta_{\text {maj }}$ and $\theta_{\text {min }}$ are the major and minor axis FWHM of the synthesized beam, respectively. 
(a) $\mathbf{R}_{21}$

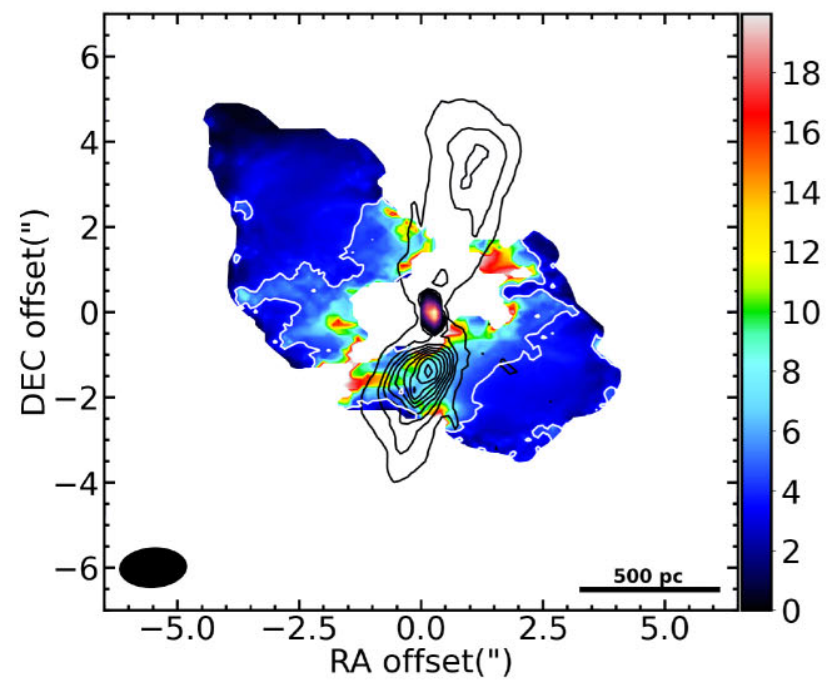

(b) $\mathbf{R}_{31}$

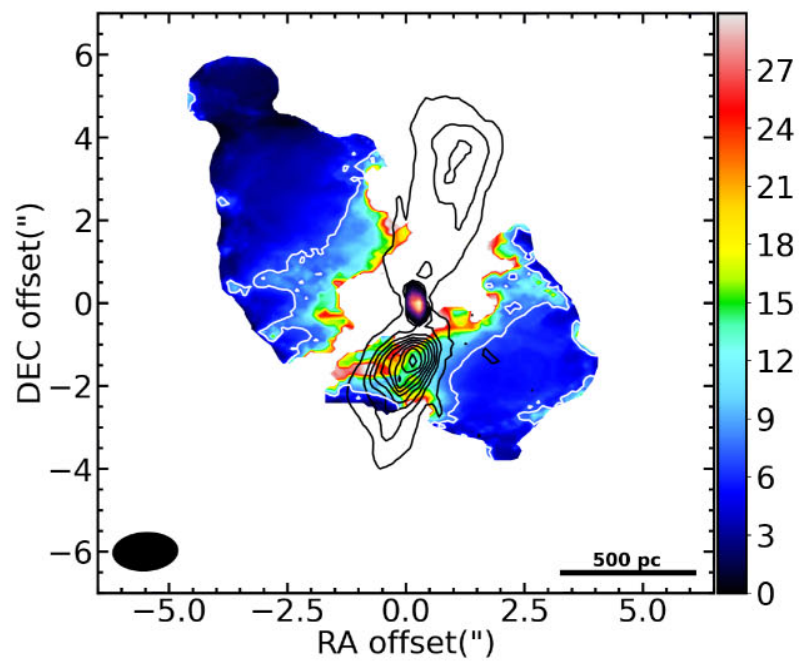

Figure 6. $R_{21} \equiv S_{\mathrm{CO}(2-1)} / S_{\mathrm{CO}(1-0)}$ (left-hand panel) and $R_{31} \equiv S_{\mathrm{CO}(3-2)} / S_{\mathrm{CO}(1-0)}$ (right-hand panel) maps with 10-GHz continuum contours overlaid. The white contour in each map roughly marks the transition between the outer low-excitation component with $R_{21}<4$ and $R_{31}<9$, and the inner high-excitation component with $R_{21} \gtrsim 4$ and $R_{31} \gtrsim 9$. The latter correspond to brightness temperature ratios $>1$ and thus likely to an optically thin gas regime (see the text for details). The bar to the right of each map shows the colour scale. In both panels, the beam size and the scale bar are shown in black in the bottom-left and bottom-right corners, respectively. Coordinates are given as relative to the image phase centre; East is to the left and North to the top.

making a spatial comparison between the regions where the highest line ratios are observed and the location of the radio lobes, it is clear that also in NGC 3100 the expanding radio plasma is likely responsible for such different gas conditions in the inner areas. More in general, it is clear from Fig. 6 that the gas becomes progressively less excited at increasing distance from the compact radio structure.

The $\mathrm{HCO}^{+}$(4-3) line requires much larger gas densities to be excited $\left(n_{\text {crit }}>1.8 \times 10^{6} \mathrm{~cm}^{-3}\right.$; Greve et al. 2009), compared to $\mathrm{CO}$ transitions $\left(n_{\text {crit }}>2.1 \times 10^{3} \mathrm{~cm}^{-3}\right.$; Carilli \& Walter 2013). As such, comparing their relative strength can provide clues on the gas density conditions in the region where they are cospatial. It is therefore interesting to analyse also the $\mathrm{HCO}^{+} / \mathrm{CO}$ flux density ratio, even though we detected the $\mathrm{HCO}^{+}(4-3)$ emission at much lower level and in a substantially smaller region than the CO transitions. To avoid unnecessary repetitions and - at the same time - allow a coherent comparison with $\mathrm{HCO}^{+} / \mathrm{CO}$ ratios reported in other studies of jet-ISM interactions (e.g. Oosterloo et al. 2017), we made a sole representative $\mathrm{R}_{\mathrm{HCO}} \equiv S_{\mathrm{HCO}^{+}(4-3)} / \mathrm{S}_{\mathrm{CO}(2-1)}$ map. We used the same method described above but binned the $\mathrm{CO}(2-1)$ data cube to a channel width of $105 \mathrm{~km} \mathrm{~s}^{-1}$. The resulting $\mathrm{R}_{\mathrm{HCO}}$ map is shown in Fig. 7 with $10-\mathrm{GHz}$ continuum contours overlaid in black. The $\mathrm{R}_{\mathrm{HCO}}$ values are found to vary from $\approx 0.1$ to $\approx 0.7$, much higher than those typically observed in unperturbed gas regions (<0.045; e.g. Oosterloo et al. 2017). Enhanced dense gas emission can be induced by various types of perturbations (such as merger-induced shocks; see e.g. König et al. 2018). However, Fig. 7 clearly shows that the faint $\mathrm{HCO}^{+}(4-3)$ emission arises from a region adjacent to the base of the southern radio lobe, making a jet-induced gas compression the most likely scenario.

In general, all the results presented above provide important constraints on the radio jet-cold gas interplay in NGC 3100. This is discussed in detail Section 5.2.

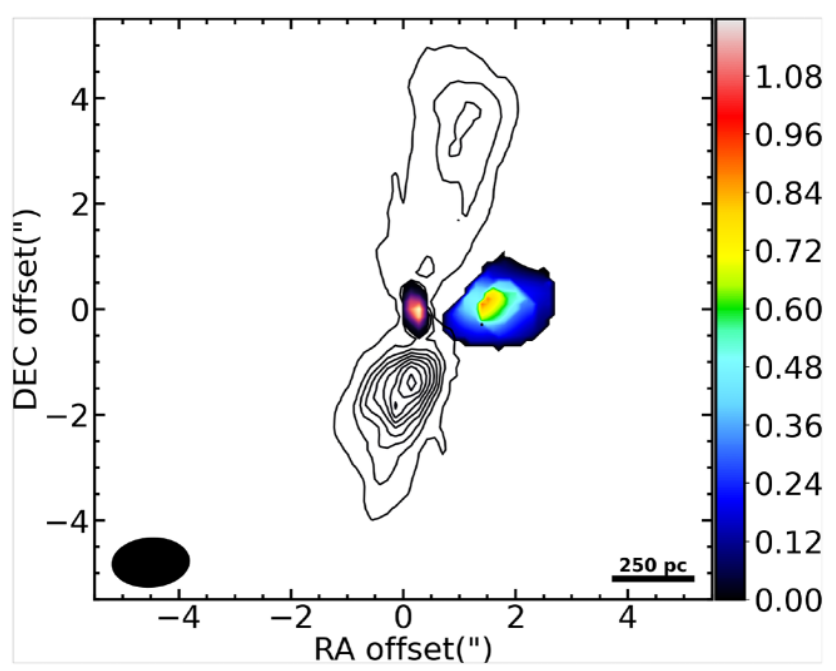

Figure 7. $\mathrm{R}_{\mathrm{HCO}} \equiv \mathrm{S}_{\mathrm{HCO}^{+}(4-3)} / S_{\mathrm{CO}(2-1)}$ map with $10-\mathrm{GHz}$ continuum contours overlaid. The bar to the right shows the colour scale. The beam size and the scale bar are shown in black in the bottom-left and bottom-right corners, respectively. Coordinates are given as relative to the image phase centre; East is to the left and North to the top.

\subsection{CO kinematics}

A detailed study of the molecular gas kinematics in NGC 3100 was presented in Paper II, based on our previous $\mathrm{CO}(2-1)$ observations. Among other interesting results, this analysis demonstrated the presence of radial motions (estimated to be $\leq 10$ per cent of the underlying rotational velocity) in the plane of the $\mathrm{CO}$ disc. We tentatively identified these as inflow streaming motions likely induced by the two-armed spiral perturbation that we found in the inner $\mathrm{CO}$ regions (see Paper II for details). The hole at the centre of the $\mathrm{CO}(2-1)$ 
distribution, however, made these results somewhat uncertain. It is thus interesting to repeat the same analysis on the $\mathrm{CO}(3-2)$ line, which is the sole among the observed $\mathrm{CO}$ transitions tracing the gas up to the very centre of the source. The procedure adopted to carry out the kinematic modelling is the same as that followed for the $\mathrm{CO}(2-1)$ transition. This was extensively described in Paper II; hence, only an outline is provided in the following.

We analysed the CO kinematics using the INTEGRATED DEVELOPMENT LANGUAGE (IDL) version of the KinEMATIC MOLECULAR SiMULATION tool (KINMS $;{ }^{5}$ Davis et al. 2013). This routine allows to input arbitrary functions describing the gas distribution and kinematics and constructs the corresponding mock data cube by calculating the line-of-sight projection of the circular velocity for a defined number (typically $10^{5}-10^{6}$ ) of point-like sources representing the gas distribution. As for the $\mathrm{CO}(2-1)$ transition, we modelled the $\mathrm{CO}(3-2)$ distribution with a nuclear two-armed spiral structure (very well reproducing the observed $\mathrm{CO}$ peaks at the SW/NE side of the core; Fig. 3, left-hand panels) embedded in a disc. The former was parametrized in KINMS as a uniform brightness distribution along loci whose positions from the phase centre are described by a logarithmic spiral function; the latter as an axisymmetric exponential disc (a form that has been shown to be appropriate in many ETGs; e.g. Davis et al. 2013). The s-shaped velocity iso-contours visible in the rotation pattern (Fig. 3, middle column) may be caused by either warps or non-circular motions, as both phenomena produce the same kinematic features. In NGC 3100, however, we found that they were best reproduced by adding both a position angle (PA) and an inclination ( $i$ ) warp (i.e. allowing a linear radial variation of both parameters between a lower and an upper value). The gas was then assumed to be in purely circular motion and its rotation curve was modelled using a simple arctangent function (see e.g. Swinbank et al. 2012; van de Voort et al. 2018). This approach still allows to identify possible non-circular motions, if significant residuals (i.e. larger than the data cube channel width) are found once such simple rotating model is subtracted from the observed velocity field. We also included in our model a velocity dispersion parameter $\left(\sigma_{\text {gas }}\right)$. Since our aim is simply to obtain an average value for the intrinsic gas velocity dispersion across the gas disc, $\sigma_{\text {gas }}$ was assumed to be spatially constant. The so-simulated data cube was then fitted to the observed one using the Markov Chain Monte Carlo (MCMC) code KINMS_MCMC, ${ }^{6}$ which explores the full space defined by the input model and outputs the full Bayesian posterior probability distribution, identifying the best-fitting set of parameters. These latter are fully in agreement with that obtained from the $\mathrm{CO}(2-$ 1) kinematic modelling and thus not further discussed here (see Table 6 and Paper II for details). We only note that the best-fitting $\sigma_{\text {gas }}$ was found to vary from $\approx 34 \pm 4$ to $\approx 41 \pm 3 \mathrm{~km} \mathrm{~s}^{-1}$ from the $2-1$ to the 3-2 transition. ${ }^{7}$ Although still consistent within their respective errors, these two values imply that the average gas velocity dispersion is intrinsically large, thus confirming the scenario inferred from the visual inspection of the line-of-sight mean velocity dispersion maps (i.e. the dynamical state of the cold gas can be considered turbulent on average; see Section 4.1).

The best-fitting $\mathrm{CO}(3-2)$ mean line-of-sight velocity map shown in Fig. 8(a) illustrates that our simple rotating model provides a good

\footnotetext{
${ }^{5}$ https://github.com/TimothyADavis/KinMS

${ }^{6}$ https://github.com/TimothyADavis/KinMS_MCMC

${ }^{7}$ Due to a more accurate processing of the uncertainties in the $\chi^{2}$ statistics, the error associated to the best-fitting $\mathrm{CO}(2-1) \sigma_{\text {gas }}$ reported here is larger than that tabulated in Paper II.
}

Table 6. Best-fitting parameters from the kinematic modelling of the $\mathrm{CO}(3-$ 2) transition.

\begin{tabular}{lccc}
\hline ID & Parameter & Unit & Value \\
\hline$(1)$ & Kinematic position angle, PA & $(\mathrm{deg})$ & $190-250$ \\
$(2)$ & Disc inclination, $i$ & $(\mathrm{deg})$ & $20-80$ \\
$(3)$ & Disc scale length, $\mathrm{R}_{\text {disc }}$ & $(\operatorname{arcsec})$ & $3.1 \pm 0.3$ \\
$(4)$ & Maximum circular velocity, $v_{\text {flat }}$ & $\left(\mathrm{km} \mathrm{s}^{-1}\right)$ & $345 \pm 5$ \\
$(5)$ & Rotation curve turn over radius, $r_{\text {turn }}$ & $(\operatorname{arcsec})$ & $2.6 \pm 0.2$ \\
$(6)$ & Velocity dispersion, $\sigma_{\text {gas }}$ & $\left(\mathrm{km} \mathrm{s}^{-1}\right)$ & $41 \pm 3$ \\
\hline
\end{tabular}

Note. Rows: (1) Range of the $\mathrm{CO}$ disc kinematic position angle (i.e. the PA measured counterclockwise from North to the approaching side of the velocity field). (2) Range of the CO disc inclination. (3) Scale length of the CO disc surface brightness profile. (4) Asymptotic (or maximum) circular velocity in the flat part of the CO rotation curve (corresponding to the major axis PVD shown in Fig. 8c). (5) Effective radius at which the $\mathrm{CO}$ rotation curve turns over. (6) Gas velocity dispersion.

overall representation to the observed kinematics (Fig. 3c, middle panel). The distortions in the rotation pattern are also reasonably well reproduced by the combination of warps in both PA and inclination included in the model, in agreement with what found from the $\mathrm{CO}(2-1)$ modelling. Our main aim here, however, is to look for kinematic signatures that can confirm and/or complement our previous finding of non-circular motions in the plane of the $\mathrm{CO}$ disc. Particularly interesting in this regard are the mean velocity residuals visible in Fig. 8(b), with amplitudes $\leq 70 \mathrm{~km} \mathrm{~s}^{-1}$ in absolute value (or $\leq 91 \mathrm{~km} \mathrm{~s}^{-1}$ deprojected). These are slightly larger than those found in the $\mathrm{CO}(2-1)$ (see Section 2 and Paper II) and - differently from them - can be clearly divided into two main groups, each lying along a different kinematic axis. One group consists of two regions along the kinematic major axis with peaks centred at RA offsets of $\approx 2^{\prime \prime} .0$ and $1^{\prime \prime} .5$ to the NE and SW of the core, respectively, clearly spatially correlated with the positions of the two $\mathrm{CO}(3-2)$ peaks visible in Fig. 3(c) (left-hand panel). The two regions exhibit mostly redshifted/blueshifted velocities, respectively: knowing from the BI colour map of NGC 3100 (Fig. 1, top right-hand panel) that the eastern is the near side of the disc (see also Paper II), the observed residual velocities would imply gas inflow.

A second group of significant residuals can be identified in Fig. 8(b) along the kinematic minor axis. These are particularly interesting as the gas rotational component is mostly negligible along the minor axis and thus significant velocity components are likely to be ascribed to radial motions. The presence of such motions can also be clearly inferred from the minor axis PVD in Fig. 8(d), showing a gradient with velocities up to $\approx \pm 200 \mathrm{~km}^{-1}$ not reproduced by our model (overlaid in cyan). The minor axis PVD of gas in pure circular motions is indeed expected to show nearly flat emission around the systemic velocity, with typical widths of a few tens of $\mathrm{km} \mathrm{s}^{-1}$ (due to a combination of intrinsic gas velocity dispersion and beam smearing effects; e.g. García-Burillo et al. 2014). The residual velocities (both in the residual map and the minor axis PVD) are preferentially blueshifted to the SE and redshifted to the $\mathrm{NW}$, implying gas outflow when compared with the near side of the CO disc.

Caution is needed, however, as interpreting the gas motions from the sole residual map may lead to draw inaccurate conclusions. This is because - by definition - the mean line-of-sight velocities are flux density-weighted and thus biased towards the brighter components. As a further check, we then extracted spectral profiles from the cleaned $\mathrm{CO}(3-2)$ data cube within the regions enclosing the most significant velocity residuals described above. If the gas motion in 
(a) Model moment one

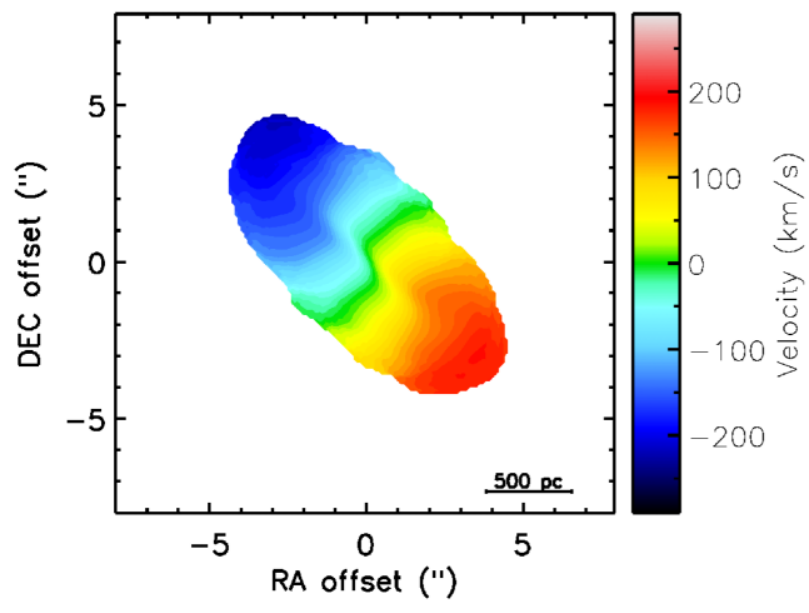

(c) Major axis PVD

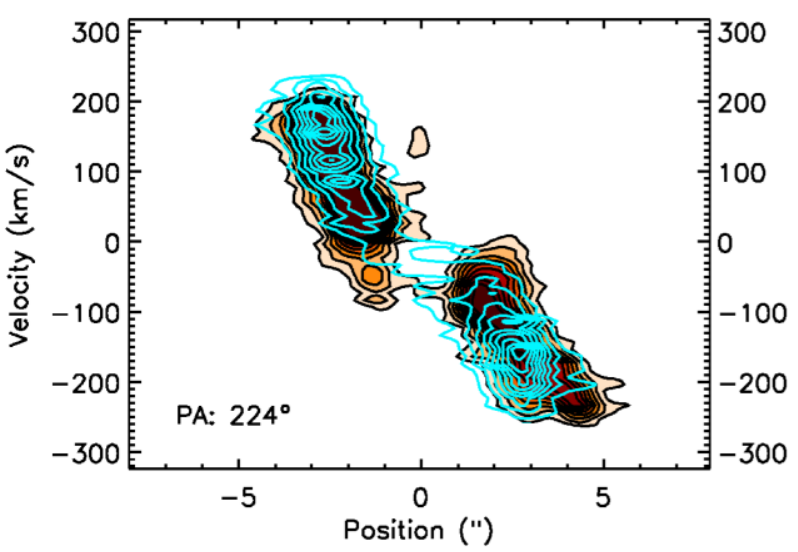

(b) Data - Model moment one

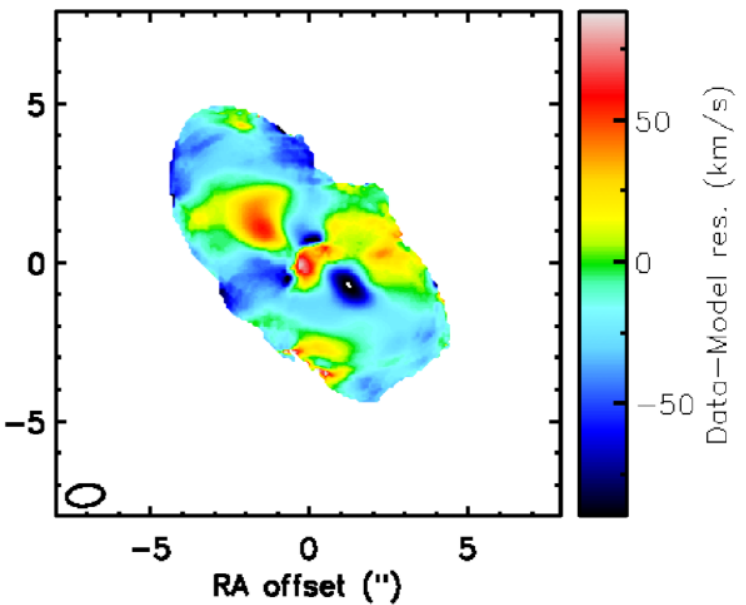

(d) Minor axis PVD

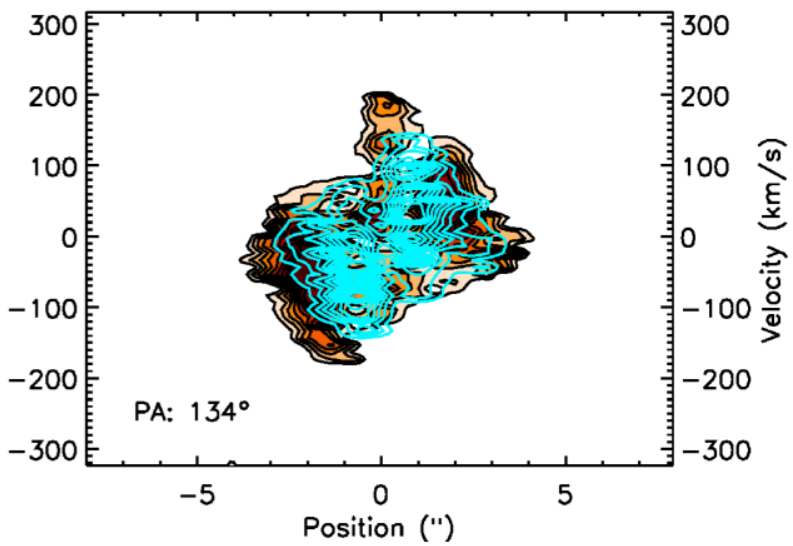

Figure 8. $\mathrm{CO}(3-2)$ model and residual (data model) mean velocity maps (panels a and $\mathrm{b}$, respectively). The bars to the right show the colour scale in $\mathrm{km} \mathrm{s}^{-1}$ East is to the left and North to the top. Velocities are measured in the source frame and the zero-point corresponds to the intensity-weighted centroid of the CO emission. The maps are created with the masked moment technique described in Section 4.1, using a data cube with a channel width of $10 \mathrm{~km} \mathrm{~s}^{-1}$. The $\mathrm{CO}(3-2)$ major and minor axis PVDs are shown in panels $\mathrm{c}$ and $\mathrm{d}$, respectively. The former is extracted within a rectangular area whose long axis is orientated according to the kinematic position angle indicated in the bottom left corner of the panel (i.e. along the CO major axis); the same rectangular area is rotated by $90^{\circ}$ to extract the PVD along the kinematic minor axis. The contours of the best-fitting model are overlaid in cyan. The contours are drawn at $3,9, \ldots$ times the $1 \sigma$ rms noise level (listed in Table 4).

the area of the spectral extraction is dominated by regular rotation, we should observe a single spectral component with a centroid close to the systemic velocity. Additional, higher-velocity spectral components are likely related to local gas flows and indicate departures from pure circular rotation (see e.g. Domínguez-Fernández et al. 2020). The obtained spectral profiles and corresponding regions of extraction are illustrated in Fig. 9.

In all the cases, we can clearly distinguish multiple kinematic components. The spectral profile in the top left corner of Fig. 9 is extracted from the area to the NE of the core with large redshifted residuals (labelled as region $\mathrm{A}$ ). This shows both a main narrow emission component tracing the local gas circular motion and a secondary component observed as a blushifted excess with velocities up to $\approx-250 \mathrm{~km} \mathrm{~s}^{-1}$. We note that this latter has exactly opposite velocities compared to those expected from just the inspection of the mean line-of-sight velocity residual map, stressing the importance of analysing the velocity residuals together with the local spectral profiles. The panel in the bottom right corner of Fig. 9 shows the spectrum of the region to the SW of the core (labelled as region $\mathrm{B}$ ) with large blueshifted residuals. In this case, both a blueshifted and a redshifted excess (with velocities up to $\approx-120$ and $\approx+90 \mathrm{~km} \mathrm{~s}^{-1}$, respectively) are observed in addition to the main narrow component. By comparing the velocities of the broader kinematic components in region $\mathrm{A}$ and $\mathrm{B}$ spectra with the near side of the disc, we conclude that they are likely tracing both inflow/outflow motions. As mentioned above, these are clearly spatially correlated with the positions of the two CO peaks, and thus - in turn - with the location of the inner spiral perturbation. Spiral shocks and the associated turbulence can induce streaming motions, causing the gas to lose or gain angular momentum and move inwards or outwards, respectively (e.g. van de Ven \& Fathi 2010). We then more robustly confirm and complement the scenario only tentatively inferred from the previous $\mathrm{CO}(2-1)$ kinematic modelling (Paper II): the non-circular motions traced by the velocity residuals at the NE and the SW of the core and along the CO kinematic major axis are likely in the form of inflow/outflow streaming motions induced by the inner spiral perturbation. 

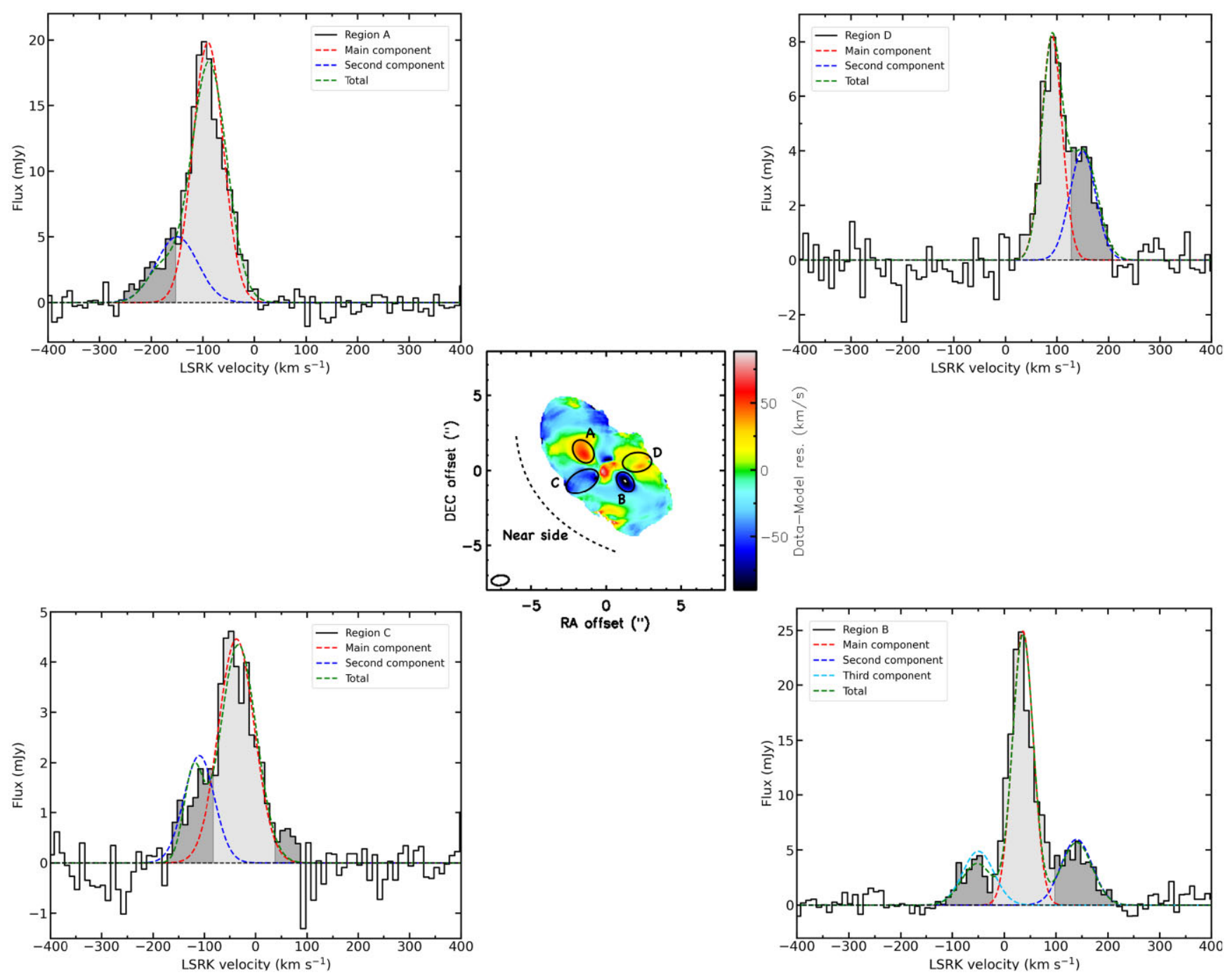

Figure 9. $\mathrm{CO}(3-2)$ spectral profiles extracted from the cleaned data cube within boxes enclosing the regions of the most significant data-model velocity residuals (central panel). The two regions to the NE and SW of the core and along the kinematic major axis are labelled as A and B, respectively; those along the kinematic minor axis are labelled as $\mathrm{C}$ and $\mathrm{D}$ (see the text for details). The curved black dashed line in the central panel marks the near side of the CO disc. In each spectral profile, the best-fitting Gaussians identifying the main, secondary, and total kinematic components are overlaid as red, blue, and green dashed lines, respectively. In the spectrum of region B (bottom right corner), the cyan dashed line illustrates the best-fitting Gaussian profile of the third additional kinematic component. The light and dark grey shaded regions highlight the spectral channels resulting as part of the main and secondary kinematic components, respectively. The black dashed horizontal line indicates the zero flux level.

The spectral profiles of the areas along the CO kinematic minor axis with the most significant velocity residuals (labelled as regions $\mathrm{C}$ and D) are shown in the bottom left and top right corners of Fig. 9, respectively. ${ }^{8}$ They show blueshifted (C) and redshifted (D) excesses with velocities up to -170 and $+200 \mathrm{~km} \mathrm{~s}^{-1}$, respectively, in addition to the main kinematic component. ${ }^{9}$ Given the orientation of the gas disc with respect to our line of sight, the observed

\footnotetext{
${ }^{8}$ We note that we also attempted to extract the spectrum of the region at the centre of the gas distribution showing the brightest redshifted residuals, but the poor $\mathrm{S} / \mathrm{N}$ ratio in that area does not allow to clearly distinguish any spectral feature and carry out a reliable analysis.

${ }^{9} \mathrm{~A}$ faint redshifted excess is also visible in the spectrum of Region C (bottom left corner of Fig. 9) and highlighted as well in a dark grey colour. However, the $\mathrm{S} / \mathrm{N}$ ratio of the corresponding channels is too low to allow a proper Gaussian fitting and thus robustly identifying it as an additional kinematic component.
}

velocity excesses are compatible with an outflow. Interestingly, the spatial comparison between the outflow region and the location of the radio lobes (see bottom right-hand panel of Fig. 1) strongly suggests that the former is jet-driven. A rough estimate of the outflowing gas mass can be obtained by calculating the flux densities of the blueshifted/redshifted kinematic components integrated over the velocity ranges highlighted by the dark-grey shaded channels in regions $\mathrm{C}$ and $\mathrm{D}$ spectra and using equation (2) to convert the integrated fluxes into molecular gas masses. We note that by comparing Figs 6 and 9, it is evident that the outflowing gas is also the gas showing the largest line ratios and thus in an optically thin regime (see Section 4.4). In such condition, the $\mathrm{CO}-$ to- $\mathrm{H}_{2}$ conversion factor, $X_{\mathrm{CO}}$, depends on the gas excitation temperature (see Bolatto et al. 2013). Assuming $T_{\mathrm{ex}}=50 \mathrm{~K}$ for an average $R_{31} \approx 12$ in the high-excitation regions, we get $X_{\mathrm{CO}}=2.5 \times 10^{19} \mathrm{~cm}^{2}\left(\mathrm{~K} \mathrm{~km} \mathrm{~s}^{-1}\right)^{-1}$ and $M_{\text {out }}=5.6 \times 10^{4} \mathrm{M}_{\odot}$. This mass is only a small fraction of the total CO mass (see Section 4.3); thus, the effect of the jet-induced 
outflow in NGC 3100 appears very localized and its impact on the host galaxy as a whole is likely to be negligible. Making the simple assumption of spherical geometry for the outflowing region, an upper limit on the mass outflow rate can also be obtained from the relation $\dot{M} \lesssim 3 v_{\max } M_{\text {out }} / R_{\max }$ (e.g. Maiolino et al. 2012), where $M_{\text {out }}$ is the mass of the outflowing gas estimated above, and $R_{\max }$ and $v_{\max }$ are the maximum extent of the outflow region and maximum outflow velocity, respectively. Assuming $R_{\max }=300$ pc (i.e. the maximum extent of regions C and D in Fig. 9) and $v_{\max }=200 \mathrm{~km} \mathrm{~s}^{-1}$, we obtain $\dot{M} \lesssim 0.12 \mathrm{M}_{\odot} \mathrm{yr}^{-1}$. This is at least one order of magnitude lower than that estimated in other known cases of $\mathrm{CO}$ outflows induced by young radio jets (e.g. García-Burillo et al. 2014; Mahony et al. 2016; Oosterloo et al. 2017), definitely indicating that - at least in terms of the induced kinematic perturbations - the jet-ISM coupling in NGC 3100 has to be much less extreme. We discuss these results in Section 5.2.

\section{DISCUSSION}

\subsection{Continuum emission}

Our multifrequency $(107,136,235$, and $348 \mathrm{GHz}) \mathrm{mm}$ continuum images show the same compact core-double lobe structure seen in the radio continuum at 5 and $10 \mathrm{GHz}$ (maximum linear size $\approx 2 \mathrm{kpc}$; see Paper I and Paper III), although emission from the extended radio structures becomes progressively fainter at increasing frequencies. The great morphological similarity between the continuum emission over this wide range of frequencies suggests that the continuum of NGC 3100 is dominated by non-thermal synchrotron emission from the core and extended radio structures up to at least $348 \mathrm{GHz}$. Thermal emission from the Rayleigh-Jeans tail of the dust component is expected to contribute to the continuum at millimetre wavelengths. We find no evidence of such contribution up to the highest frequencies imaged with ALMA (i.e. $235 \mathrm{GHz}$ and $348 \mathrm{GHz}$; see Figs 2c and d), although we cannot exclude some contribution from this mechanism to the unresolved core emission.

Fig. 10 shows the radio-(sub-)mm spectrum of NGC 3100, where different colours are used for the data points of different components: core, jet, and total (core + jet). This latter includes data from our ALMA and JVLA observations (Paper I, Paper III, and this work) and also measurements from unresolved $1.4 \mathrm{GHz}, 843$ and $408 \mathrm{MHz}$ data taken from the NASA/IPAC extragalactic data base (NED; https://ned.ipac.caltech.edu). Such combination of high- and lowresolution data in the total radio to sub-mm spectrum is possible in this case because the radio source in NGC 3100 is compact and there is no evidence from the available multifrequency radio data for emission on larger scales (see Section 2 and Paper III). Flux density measurements including emission from single components (i.e. core or jet) are instead taken only from spatially resolved and matched-resolution data (i.e. the 5- and 10-GHz VLA and all ALMA continuum data from Paper I, Paper III, and this work).

To obtain spectral indices, the data points of each component are fitted with a single power-law function (of the form $S_{v}=A v^{\alpha}$, where $S_{v}$ is the flux density as a function of frequency $v, A$ is the power-law normalization, and $\alpha$ is the spectral index) using the least-squares minimization PYTHON routine lmfit (Newville et al. 2014). The spectral index and power-law normalization are left free to vary in the fitting process. The resulting best-fitting spectral indices are $\alpha_{\text {tot }}=-0.49 \pm 0.03$ for the total continuum, $\alpha_{\text {core }}=-0.3 \pm 0.2$ for the core component, and $\alpha_{\text {jet }}=-0.73 \pm 0.01$ for the jet component.

The presence of a flatter and steeper component associated with the core and jet, respectively, was already reported in Paper I, based

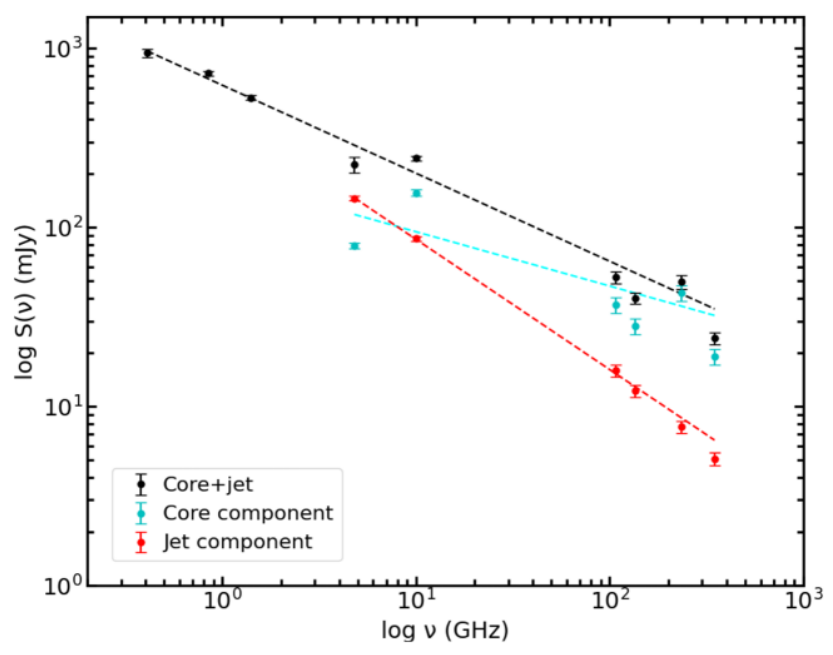

Figure 10. Radio to sub-mm spectrum of NGC 3100. Black circles are for flux density measurements encompassing emission from both the nucleus and the jets; these include data from our ALMA and JVLA observations (Paper I, Paper III, and this work) and also $1.4 \mathrm{GHz}, 843$ and $408 \mathrm{MHz}$ measurements taken from NED. Cyan and red circles are used for flux densities including emission from a single component only (core and jet, respectively); in this case, only spatially resolved and matched-resolution continuum data are considered (i.e. the 5- and 10-GHz VLA and all ALMA continuum data from Paper I, Paper III, and this work). A best-fitting power law is overlaid in the corresponding colour.

on a spatially resolved spectral index analysis carried out using the ALMA 235-GHz and VLA 5-GHz data available at that time. The obtained $\alpha_{\text {jet }}$ is consistent with optically thin synchrotron emission but is slightly steeper than that typically observed in FR I jets $(\alpha \approx$ -0.6 between 1.4 and $4.9 \mathrm{GHz}$; Laing \& Bridle 2013). Nevertheless, there is no clear sign of an abrupt high-frequency steepening due to synchrotron or inverse Compton losses. The best-fitting core spectral index, $\alpha_{\text {core }}$, is consistent with partially optically thick (self-absorbed) emission from a compact component. The observed scatter in the data points, however, results in large fitting errors, making $\alpha_{\text {core }}$ poorly constrained. Since the various flux measurements are taken at different epochs, we may be observing the effects of core variability.

In general, the radio-(sub-)mm morphological and spectral properties of the radio source in NGC 3100 (i.e. compact linear size and overall steep spectrum) support a scenario (already outlined in Paper III) in which it has likely been caught in the early phases of its evolution (see e.g. O'Dea \& Saikia 2021, for a recent review on this subject).

\subsection{Jet-cold gas interaction}

\subsubsection{CO excitation conditions and kinematics}

The analysis of the multiple CO line detections presented in this paper allows us to more robustly confirm and complement the results obtained from the sole $\mathrm{CO}(2-1)$ transition in NGC 3100 (Paper II). First of all, our analysis clearly shows that a simple model assuming gas in pure circular motions well reproduces most of the observed kinematic features, once accounting for the presence of both a PA and inclination warp (Figs 3 and 9). This confirms that the bulk of the $\mathrm{CO}$ gas in NGC 3100 is regularly rotating (at least at the spatial resolution of our ALMA observations; see Table 2). Nevertheless, a separate kinematic component can be clearly identified from the mean line-ofsight velocity residuals along the CO kinematic major axis (Fig. 9) 
and ascribed to inflow/outflow streaming motions induced by an inner two-armed spiral perturbation [already tentatively inferred from the previous $\mathrm{CO}(2-1)$ data; Paper II]. The maximum inflow/outflow velocities are $\approx+90 \mathrm{~km} \mathrm{~s}^{-1}$ and $\approx-250 \mathrm{~km} \mathrm{~s}^{-1}$, respectively (see Section 4.5 and Fig. 9). These numbers indicate that streaming motions induced by sub-kpc scale spiral shocks can be a significant fraction of the underlying circular velocity (as suggested by - e.g. - van de Ven \& Fathi 2010). As extensively discussed in Paper II, the significance of this result relies on the fact that nuclear spiral perturbations and associated non-circular motions are considered as an efficient mechanism for transporting gas from kpc scales to the nucleus and feeding the central SMBH (e.g. Combes et al. 2013; Fathi et al. 2013). Theoretical studies, however, demonstrate that nuclear spiral instabilities can produce large accretion rates (1-10 $\mathrm{M}_{\odot} \mathrm{yr}^{-1}$; e.g. Hopkins \& Quataert 2010), as opposed to the strongly sub-Eddington rates estimated in LERGs such as NGC 3100 (see below). This suggests that other physical processes intervene in the very inner regions of these objects, keeping the accretion rate low. Higher resolution observations probing the gas at scales relevant for the accretion process $(\ll 100 \mathrm{pc})$ are needed to examine the gas kinematics around the SMBH and shed light on this issue.

Beyond that, the results presented in Sections 4.4 and 4.5 clearly show that an additional ongoing perturbation is affecting both the physical conditions and kinematics of the $\mathrm{CO}$ gas located in the inner regions. Indeed, another separate kinematic component can be clearly distinguished along the $\mathrm{CO}$ kinematic minor axis and associated to an outflow (see Section 4.5 and Fig. 9). The spatial correlation between the outflow region and the location of the radio lobes strongly suggests that the former is jet-induced. The maximum observed outflow velocity is $\approx 200 \mathrm{~km} \mathrm{~s}^{-1}$ and the mass outflow rate is estimated to be $\lesssim 0.12 \mathrm{M}_{\odot} \mathrm{yr}^{-1}$ (see Section 4.5), indicating that the jet impact on global (galactic) scales is likely negligible at this stage. These values are also significantly lower than those expected from available simulations (e.g. Wagner et al. 2012, 2016; Mukherjee et al. 2016, 2018a,b) and estimated in the few existing spatially resolved studies of (sub-)kpc scale jet-cold gas interactions (e.g. Combes et al. 2013; García-Burillo et al. 2014; Morganti et al. 2015; Mahony et al. 2016; Oosterloo et al. 2017). On the other hand, the line ratios of the outflowing $\mathrm{CO}$ gas are drastically enhanced, implying optically thin conditions and high excitation temperatures ( $250 \mathrm{~K}$; see Section 4.4). Similar modifications to the CO physics have been observed in the most extreme cases of sub-kpc scale jetcold gas interactions (e.g. Oosterloo et al. 2017). In short, our results indicate that the jet-CO coupling ongoing in NGC 3100 is strongly altering the physical conditions of the involved $\mathrm{CO}$ gas fraction but only mildly affecting its kinematics.

The fact that the kinematic perturbations in the interacting $\mathrm{CO}$ gas are significantly lower compared to those expected from simulations and observations can depend on various factors. Among these, the age of the radio source may play an important role. Indeed, both simulations and observations have so far taken snapshots of the radio jet-cold gas interplay mostly during the very early phases of the jet evolution (i.e. within less than $1 \mathrm{Myr}$ from their first ignition, corresponding to linear sizes <1 kpc; e.g. Oosterloo et al. 2017; Mukherjee et al. 2018a,b; Zovaro et al. 2019). Based on observations of a handful of objects with jets at slightly different stages of their initial evolution (but no more extended than $1 \mathrm{kpc}$ ), Morganti et al. (2021) recently suggested that the impact of the radio jets may change over time. In this scenario, the kinematics of the interacting cold gas should become less perturbed as the jets break out from the core region and start to expand on larger scales (typically at ages of few Myr, corresponding to linear sizes of the radio source $\gtrsim 1 \mathrm{kpc}$ ).
The maximum linear extent of the core-double lobe radio source in NGC 3100 is $2 \mathrm{kpc}$ (see Section 5.1 and Paper III), indicating that while still relatively young - it has been caught in a more evolved phase than those explored so far. We thus consider the possibility of a causal connection between the mild kinematic perturbations observed in the $\mathrm{CO}$ gas that is interacting with the radio jets and the evolutionary stage of these latter.

As mentioned in Section 1, the jet-cold gas coupling has also been shown to be very sensitive to the jet power (Mukherjee et al. 2018b). Using well-known scaling relations between the jet and radio power (Cavagnolo et al. 2010), we estimated $P_{\text {jet }} \approx$ $1 \times 10^{43} \mathrm{erg} \mathrm{s}^{-1}$ in NGC 3100. Even though the large scatter in the adopted scaling relations makes such estimation uncertain up to one order of magnitude (see e.g. Godfrey \& Shabala 2016), this is still fully within the range of jet powers typically associated to low-power FR I radio galaxies, such as the majority of LERGs (e.g. Godfrey \& Shabala 2013). The extent that the jet-induced kinematic perturbations should have in such cases is not yet clear, as both simulations and observations have so far mostly focused on jets with powers $>10^{44} \mathrm{erg} \mathrm{s}^{-1}$ (typical of powerful radio galaxies with FR II morphologies; e.g. Godfrey \& Shabala 2016). It has been suggested that the jet-ISM coupling should be most pronounced for jets with intermediate powers $\left(P_{\text {jet }} \sim 10^{43}-10^{44} \mathrm{erg} \mathrm{s}^{-1}\right)$ lying close to the CO plane, such as in the case of NGC 3100 (see Paper III). The observed kinematic features, however, do not seem to support this hypothesis.

In addition to all the above, the few objects in which jetdriven (sub-)kpc scale CO outflows have been spatially resolved are also radiatively efficient (Seyfert-like) AGN, with typical AGN bolometric luminosities between 1 and 10 per cent of their Eddington luminosities (i.e. $L_{\mathrm{Bol}} / L_{\mathrm{Edd}} \approx 0.01-0.1$ ). Thus, as noted by Morganti et al. (2021), there is an additional source of energy in the central regions of these objects and - although primarily jet-induced - the observed cold gas perturbations may result from a combination of processes. The nuclear luminosity in NGC 3100 is strongly subEddington, ${ }^{10}$ with $L_{\mathrm{Bol}} / L_{\mathrm{Edd}}=3 \times 10^{-4}$. This is well within the values typically associated to radiatively inefficient AGN and compatible with advection-dominated accretion flows (ADAF) and its variants (e.g. Narayan et al. 2002), suggesting that the observed CO kinematic perturbations in NGC 3100 are exclusively induced by the (low) jet kinetic power.

Appropriate models, along with a statistically significant number of spatially resolved studies of jet-cold gas interactions covering wide ranges of jet ages/powers and nuclear luminosities, would be crucial to better understand the physics underlying the observed $\mathrm{CO}$ kinematics and draw more solid conclusions.

\subsubsection{Dense and shock gas tracers}

As shown in Fig. 4, the faint $\mathrm{HCO}^{+}(4-3)$ detection is restricted to a single unresolved blob-like structure at $\approx 2^{\prime \prime}$ west of the nucleus.

\footnotetext{
${ }^{10}$ We estimated the AGN bolometric luminosity in NGC 3100 using the [OIII] $\lambda 5007$ line as a proxy and applying the bolometric correction $L_{\text {bol }} / L_{[\text {OIII] }} \approx 3500$ (Heckman et al. 2004). Based on the optical spectroscopy presented by Dopita et al. (2015), $L_{[\mathrm{OIII}]} \approx 1.7 \times 10^{39} \mathrm{erg} \mathrm{s}^{-1}$ and thus $L_{\text {bol }}=$ $6 \times 10^{42} \mathrm{erg} \mathrm{s}^{-1}$. The mass of the SMBH in NGC 3100 was estimated using the scaling relation $\log \left(M_{\mathrm{SMBH}} / M_{\odot}\right)=8.23+3.96 \log \left(\sigma_{*} / 200 \mathrm{~km} \mathrm{~s}^{-1}\right)$ (Tremaine et al. 2002), where we assumed a stellar velocity dispersion $\sigma_{*}=$ $191 \mathrm{~km} \mathrm{~s}^{-1}$ taken from the HyperLeda data base (http://leda.univ-lyon1.fr). This results in $M_{\mathrm{SMBH}}=1.6 \times 10^{8} \mathrm{M}_{\odot}$ and $L_{\mathrm{Edd}}=2 \times 10^{46} \mathrm{erg} \mathrm{s}^{-1}$.
} 
A qualitative comparison with the $\mathrm{CO}(3-2)$ distribution (overlaid as blue-to-warm contours in Fig. 4) shows that the $\mathrm{HCO}^{+}(4-3)$ is not spatially correlated with the peak of the $\mathrm{CO}$ emission corresponding to the south-western spiral arm, where the gas is expected to be compressed and the density enhanced (e.g. van de Ven \& Fathi 2010; see also see Paper II). Fig. 7 instead shows that the faint $\mathrm{HCO}^{+}(4-3)$ emission arises from a region adjacent to the base of the northern radio lobe, co-spatial with some of the largest $R_{21}$ and $R_{31}$ values (Fig. 6) and the redshifted outflow region (Fig. 9). This suggests a correlation between the jet-ISM coupling and the detected $\mathrm{HCO}^{+}$ emission. Indeed, 3D hydrodynamical simulations show that the jet plasma expanding through the surrounding gaseous matter inflates pseudo-spherical bubbles driving shocks that can not only accelerate the gas outwards but also heat (up to kinetic temperatures of $\approx 100 \mathrm{~K}$ ) and compress it, yielding to enhanced dense gas emissions up to distances a few kpc away from the main jet/lobe axis (e.g. Mukherjee et al. 2016, 2018b; Mukherjee, Bicknell \& Wagner 2021). Also, the $\mathrm{R}_{\mathrm{HCO}}$ flux density ratio seems to confirm this scenario, reaching peaks of $\approx 0.7$ (Fig. 7). These are consistent with those observed in regions of jet-ISM interactions and much larger than those typical of unperturbed gas regions $(<0.045 ;$ e.g. Oosterloo et al. 2017). Another interesting aspect to consider is that $\mathrm{HCO}^{+}$emission is detected only at blueshifted velocities (with a peak at $\approx-200 \mathrm{~km} \mathrm{~s}^{-1}$; see Fig. 5b), while it overlaps with a region located on the receding side of the CO disc (Fig. 4). This suggests that the dense and tenuous gas components are kinematically decoupled and may indicate that the former is tracing gas that is deviating from regular rotation (e.g. Young et al. 2021).

As described in Section 1, the $\mathrm{SiO}$ and HNCO molecules are commonly considered good tracers of shocks, with $\mathrm{HNCO}(6-5) / \mathrm{SiO}(3-$ 2) flux density ratios (i.e. the ratios of the transitions targeted in our ALMA observations) observed to vary from $\approx 0.3$ to $\approx 3$ from fast $\left(v>50 \mathrm{~km} \mathrm{~s}^{-1}\right)$ to slow $\left(v<20 \mathrm{~km} \mathrm{~s}^{-1}\right)$ shocks, respectively (e.g. Kelly et al. 2017). Strong $\mathrm{SiO}$ emission, in particular, has been detected in regions co-spatial with the radio jets and interpreted as an evidence of a jet-ISM interaction (e.g. García-Burillo et al. 2010). On the other hand, HNCO emission can be also observed without the need of a shock, as it has been clearly detected in unperturbed regions of nearby ETGs (e.g. Topal et al. 2016). Our analysis shows that both conditions (i.e. perturbed and unperturbed) co-exist in the cold gas component of NGC 3100. Therefore, in this context, the non-detection of any of these two transitions is surprising. One possibility is that such non-detections, together with the faint $\mathrm{HCO}^{+}(4-3)$ detection, trace an intrinsic deficiency of very dense $\left(n_{\text {crit }} \gg 10^{6} \mathrm{~cm}^{-3}\right)$ cold gas in this object. This hypothesis, however, currently remains purely speculative, as observations of other $\mathrm{HCO}^{+}$transitions and/or of different dense/shocks gas tracers (such as $\mathrm{HCN}, \mathrm{HNC}, \mathrm{CS}, \mathrm{CH}_{3} \mathrm{OH}$, etc.) would be needed to draw more solid conclusions.

\subsection{CO-dust comparison and gas-phase metallicity: implications for the gas origin}

The spatial correspondence between dust and molecular gas is generally interpreted as indicating that both trace the same ISM component (e.g. Alatalo et al. 2013). In Paper I, we demonstrated the tight co-spatiality between the central dust and ring-like $\mathrm{CO}(2-1)$ structures (see also the bottom right-hand panel of Fig. 1). Given its larger-scale sky distribution, it is interesting to repeat such comparison for the 1-0 transition. The $\mathrm{CO}(1-0)$ integrated intensity contours are overlaid on the $B-I$ colour map of NGC 3100 in Fig. 11. This image clearly shows that not only the inner gas and dust

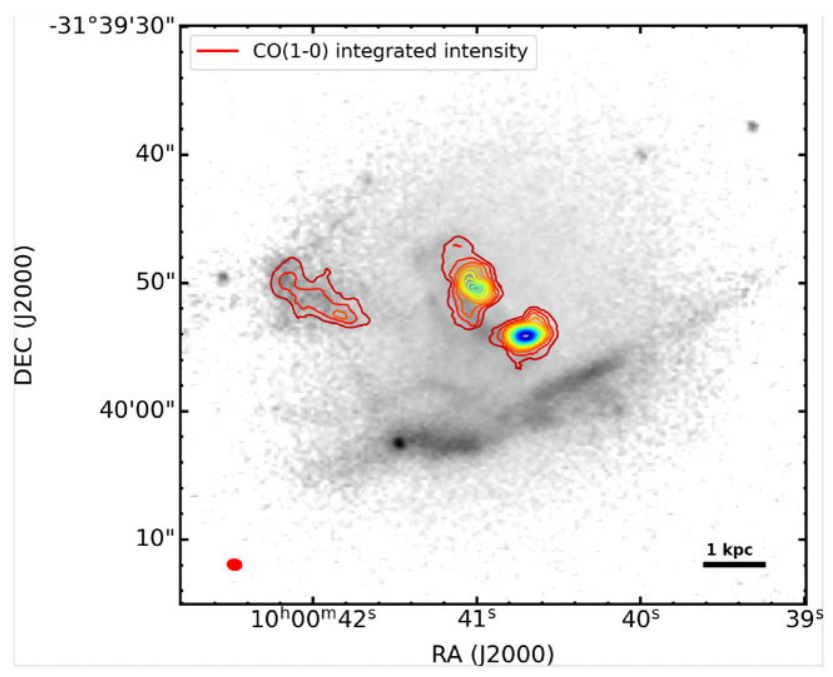

Figure 11. $C O(1-0)$-integrated intensity contours overlaid to the $B-I$ dust extinction map of NGC 3100 (see Paper I) zoomed in the central $50^{\prime \prime} \times 50^{\prime \prime}$ $\left(\sim 9 \times 9 \mathrm{kpc}^{2}\right)$. CO contours are drawn at 1,3,9 $\ldots$ times the $3-\sigma$ rms noise level. The CO beam size and scale bar are shown in the bottom left and bottom right corners, respectively; East is to the left and North to the top.

structures are spatially coincident but also the eastern $\mathrm{CO}$ patch accurately traces that of the dust. Curiously, the prominent southern dust lane is not matched by any CO emission, at least at the spatial resolution and sensitivity of our $\mathrm{CO}$ observations.

The detection of significant amounts of dust and molecular gas at the centre of nearby ETGs - the typical hosts of LERGs - points towards a recent ISM regeneration (Young et al. 2014). The origin of this material, however, is still unclear (e.g. Davis \& Bureau 2016): it may be either internally generated (from stellar mass loss and/or cooling from the hot halo) or externally accreted (from interactions or minor mergers). Disentangling these two scenarios is crucial for fully understanding the powering mechanism of the AGN in LERGs, which is still a matter of debate (e.g. Hardcastle 2018). This issue was extensively discussed in Paper II. In summary, both theoretical and observational evidence suggest that the cold gas in LERGs in rich groups and clusters (where they are preferentially located) originates from their hot atmospheres, either directly and smoothly (e.g. Lagos et al. 2015) or after chaotic cooling (as predicted in the so-called chaotic cold accretion models; e.g. Nayakshin, Power \& King 2012; Gaspari, Ruszkowski \& Oh 2013; King \& Nixon 2015). External accretion (mostly in the form of interaction with neighbouring galaxies) has been instead proposed as the dominant mechanism for the gas supply in early-type hosts in poor environments (e.g. Sabater, Best \& Heckman 2015; Davis et al. 2019; Storchi-Bergmann \& SchnorrMüller 2019), such as the majority of the LERGs in our sample (see Paper II). Exploring various observational and theoretical constraints [such as the (mis-)alignment between the $\mathrm{CO}$ and the stellar rotation axes, the presence of warps, distortions and lopsidedness in the gas morphology and kinematics, etc.], we found that an external gas origin is strongly favoured in sample sources with close companions, including NGC 3100 (see Section 2). The finding of a tight dustmolecular gas co-spatiality has also important implications in this regard, as the cold gas and dust in NGC 3100 are clearly still in the process of settling into the host galaxy potential and 'freshly' cooling cold gas structures are both predicted (e.g. Mathews \& Brighenti 2003; Hirashita \& Nozawa 2017) and observed (e.g. Lim, Ao \& Dinh-V-Trung 2008; Russell et al. 2016; Temi et al. 2018) to be 
essentially dustless (with typical gas-to-dust mass ratios of $\approx 10^{5}$; e.g. Valentini \& Brighenti 2015).

Estimating the gas-phase metallicity in the regions where dust and gas are co-spatial can provide further constraints in this regard. Indeed, dusty cold gas produced through internal processes (i.e. stellar mass loss and/or hot halo cooling) in ETGs is expected to have a higher abundance of heavy elements (i.e. super-solar metallicities) than ISM acquired from outside the galaxy (e.g. Martini, Dicken \& Storchi-Bergmann 2013; Davis et al. 2015). Following Draine et al. (2007), we estimated the gas-phase metallicity in the central dusty gas structure and eastern patch (i.e. in the regions where the $\mathrm{CO}$-dust spatial correlation is observed in Fig. 11) as:

$12+\log _{10}(\mathrm{O} / \mathrm{H})=12+\log _{10}\left(\frac{4.57088 \times 10^{-2}}{M_{\text {gas }} / M_{\text {dust }}}\right)$.

The numerical factor is set as to obtain the solar metallicity $(12+$ $\log _{10}(\mathrm{O} / \mathrm{H})=8.66$; Asplund et al. 2004) at a gas-to-dust mass ratio of $100 . M_{\text {gas }} / M_{\text {dust }}$ is the gas-to-dust mass ratio in the region(s) of interest, and $M_{\text {gas }}$ refers to the total gas mass $\left(M_{\mathrm{HI}}+M_{\mathrm{H}_{2}}\right)$. Spatially resolved observations of the atomic hydrogen in NGC 3100 are currently not available. We thus estimate a molecular gas-to-dust mass ratio, which thus yields to an upper limit of the actual gasphase metallicity. Based on the works of (e.g.) Kaviraj et al. (2012), the mass of dust clumps and patches can be estimated as:

$M_{\text {dust }}=\Sigma\left\langle A_{\lambda}\right\rangle \Gamma_{\lambda}^{-1}$,

where $\Sigma$ is the area covered by the dust features, $\left\langle A_{\lambda}\right\rangle$ is the wavelength-dependent mean extinction (i.e. dust absorption) of that area (measured directly from the $B-I$ colour map and equal to 2.0 and 1.7 mag in the central structure and eastern patch, respectively), and $\Gamma_{\lambda}$ is the mass absorption coefficient at the observed wavelength. Assuming that the dust properties of ETGs are similar to those of our Galaxy (e.g. Finkelman et al. 2008), we adopt the Milky Way $B$-band

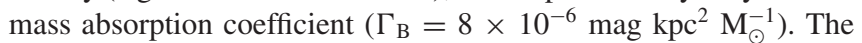
estimated dust mass in the regions co-spatial with the molecular gas is then $2.5 \times 10^{5} \mathrm{M}_{\odot}$. The molecular gas mass in the same areas is $1.4 \times 10^{8} \mathrm{M}_{\odot}$. These values imply a molecular $M_{\text {gas }} / M_{\text {dust }}=560$ and a gas-phase metallicity $<7.9$, which is about six times smaller than the solar metallicity. Such high molecular gas-to-dust mass ratio at low metallicity is consistent with that measured in galaxies within the DustPedia sample (Casasola et al. 2020) and when measured in ETGs is interpreted as an indication of external gas accretion (e.g. Kaviraj et al. 2012; Davis et al. 2015). It is worth noting, however, that a major uncertainty in the estimated gas-phase metallicity is introduced by the molecular gas mass and thus by our assumption on the $X_{\mathrm{CO}}$ conversion factor, which is in turn expected to vary with metallicity (see Section 4.3). A reliable independent estimate of the gas-phase metallicity is currently not available for NGC 3100. It is nevertheless possible to qualitatively argue that even accounting for the largest $X_{\mathrm{CO}}$ variations, the above result remains unchanged. This is trivial for $X_{\mathrm{CO}}$ larger than the Milky Way value (as typically expected in low-metallicity regions; see Bolatto et al. 2013) and will be thus no further discussed. If we instead take $X_{\mathrm{CO}}$ at the lowest end of the assumed uncertainty range (see Section 4.3), we obtain a molecular gas-to-dust mass ratio $M_{\text {gas }} / M_{\text {dust }} \approx 200$ and then a gasphase metallicity, which is about 2.5 times smaller than the solar one. We thus conclude that (at least the bulk of) the cold gas and dust in NGC 3100 originate from the interaction with the close gas-rich latetype companion, NGC 3095. This scenario is further supported also by recently acquired neutral hydrogen (HI) observations showing the presence of cold gas filaments in the field between the two companions (Maccagni et al., in preparation).

\section{SUMMARY}

This is the fourth paper of a project aimed at undertaking an extensive multiphase study of a complete, volume- and flux-limited $(z<$ $0.03, \mathrm{~S}_{2.7 \mathrm{GHz}} \geq 0.25 \mathrm{Jy}$ ) sample of 11 LERGs in the southern sky. In this paper, we presented follow-up ALMA observations of one sample source, NGC 3100, targeting the ${ }^{12} \mathrm{CO}(1-0)$ and ${ }^{12} \mathrm{CO}(3-2)$ transitions, along with the dense $\mathrm{HCO}^{+}(4-3)$ and shock $\mathrm{SiO}(3-2)$ and $\mathrm{HNCO}(6-5)$ cold gas tracers. These data complemented previously acquired ${ }^{12} \mathrm{CO}(2-1)$ and mm-radio continuum observations of NGC 3100 and allowed us to primarily carry out a detailed analysis of the molecular gas conditions and jet-ISM interaction in this object.

The main results can be summarized as follows:

(i) Mm-radio continuum: The multifrequency (107, 136, 235, and $348 \mathrm{GHz}$ ) mm continuum images of NGC 3100 show the same compact core-double lobe structure seen in the radio continuum at 5 and $10 \mathrm{GHz}$ (maximum linear size $\approx 2 \mathrm{kpc}$ ), suggesting that the continuum emission is dominated by non-thermal synchrotron emission up to at least $348 \mathrm{GHz}$. By fitting the radio-(sub-)mm spectrum, we obtain best-fitting spectral indices $\alpha_{\text {tot }}=-0.49 \pm 0.03, \alpha_{\text {core }}=$ $-0.3 \pm 0.2$, and $\alpha_{\text {jet }}=-0.73 \pm 0.01$ for the total (core + jet), core and jet components, respectively. This confirms that the observed continuum arises mainly from synchrotron emission (self-absorbed in the core and optically thin in the jets). In addition, the radio to (sub-)mm morphological and spectral continuum properties (i.e. compact linear size and overall steep spectrum) are consistent with those of a radio galaxy in the early phases of its evolution.

(ii) CO morphology: ${ }^{12} \mathrm{CO}(1-0)$ and ${ }^{12} \mathrm{CO}(3-2)$ lines are nicely detected and complement our previous ${ }^{12} \mathrm{CO}(2-1)$ observations. The integrated intensity maps of these lines show that in all the cases, the bulk of the emission arises from two bright peaks to the NE and the SW of the core, already identified from the previous ${ }^{12} \mathrm{CO}(2-1)$ analysis as the loci of a two-armed spiral structure. This is surrounded by fainter emission extending up to $\approx 2 \mathrm{kpc}$ along the major axis. In general, substantial differences are observed in the morphology of the three lines: for instance, a gap is observed at the centre of the main $\mathrm{CO}(1-0)$ distribution, whereas the $\mathrm{CO}(2-1)$ and $\mathrm{CO}(3-2)$ lines show a ring-like and full disc morphology, respectively. This suggests higher gas excitation in the central regions.

(iii) $\mathrm{CO}$ excitation conditions: The analysis of the $R_{21} \equiv$ $S_{\mathrm{CO}(2-1)} / S_{\mathrm{CO}(1-0)}$ and $R_{31} \equiv S_{\mathrm{CO}(3-2)} / S_{\mathrm{CO}(1-0)}$ flux density ratios confirms the presence of two distinct gas components: an outer low-excitation component with line ratios mostly between $\approx 1$ and $\approx 5$, and an inner high-excitation component with $R_{21} \geq 9$ and $R_{31} \geq 12$. The latter is consistent with gas in an optically thin regime and excitation temperatures $T_{\mathrm{ex}} \gtrsim 50 \mathrm{~K}$. Such conditions are very similar to those observed in the few existing spatially resolved, multiline studies of jet-ISM interactions. The spatial correlation between the regions where the largest line ratios are observed and the location of the radio lobes suggests that also in NGC 3100, the expanding radio plasma is likely responsible for the extreme gas conditions in the circumnuclear regions.

(iv) CO kinematics: The mean line-of-sight velocity maps of the three $\mathrm{CO}$ lines show that the gas is rotating but with evident kinematic distortions indicating the presence of unrelaxed substructures in the gas distribution. The accurate $3 \mathrm{D}$ kinematic modelling of the $\mathrm{CO}(3-$ 2) transition allows us to more robustly confirm and complement the results obtained from the same analysis of the sole $\mathrm{CO}(2-1)$ transition: the bulk of the cold gas is regularly rotating. Nevertheless, two distinct non-rotating kinematic components can be identified in the central gas regions. The first is observed along the $\mathrm{CO}$ kinematic major axis and can be ascribed to inflow/outflow streaming motions (with maximum velocities of $\approx+90 \mathrm{~km} \mathrm{~s}^{-1}$ and $\approx-250 \mathrm{~km} \mathrm{~s}^{-1}$, 
respectively) induced by the inner spiral perturbation. The second is observed along the kinematic minor axis and can be associated to a jet-induced outflow with $v_{\max } \approx 200 \mathrm{~km} \mathrm{~s}^{-1}$ and $\dot{M} \lesssim 0.12 \mathrm{M}_{\odot} \mathrm{yr}^{-1}$. These values suggest that the impact of the jet-ISM coupling on the host galaxy is likely negligible at this stage. The outflow kinematic properties are also significantly less extreme than those expected from jet feedback models and observed in other cases of sub-kpc scale jet-cold gas interactions. Such discrepancy may be explained in terms of the evolutionary stage of the radio source, jet power, and radiative efficiency of the accretion process.

(v) Dense and shock gas tracers: Emission from the dense gas tracer $\mathrm{HCO}^{+}(4-3)$ is tentatively detected (peak $\mathrm{S} / \mathrm{N}=5$ ) and consists of a single unresolved structure at $2^{\prime \prime}$ west of the nucleus. Such faint emission arises from a region adjacent to the base of the northern radio lobe, where also some of the largest $R_{21}$ and $R_{31}$ values and the jet-induced outflow are observed. This suggests that the detected $\mathrm{HCO}^{+}$emission traces a region of jet-induced gas compression. The $\mathrm{HCO}^{+}(4-3) / \mathrm{CO}(2-1)$ flux density ratio seems consistent with this scenario, reaching peaks of $\approx 0.7$ that are similar to those typically observed in regions of jet-ISM interactions. The current $\mathrm{HCO}^{+}$detection, however, is too marginal to carry out a detailed analysis and draw solid conclusions. The $\mathrm{SiO}(3-2)$ and $\mathrm{HNCO}(6-5)$ shock tracers are not detected. This is surprising, given the obvious perturbations affecting the inner regions of NGC 3100, but - along with the tentative $\mathrm{HCO}^{+}$detection - may be consistent with an intrinsic deficiency of very dense (i.e. $n_{\text {crit }}>10^{6} \mathrm{~cm}^{3}$ ) gas in the inner regions of NGC 3100.

(vi) Cold ISM origin: The CO-dust comparison demonstrates a tight co-spatiality, indicating that they trace the same ISM component. By measuring the molecular gas-to-dust ratio in the regions where they are co-spatial, we estimate a strongly sub-solar gasphase metallicity (i.e. $<7.9$ ). This is incompatible with gas produced through internal processes (i.e. stellar mass loss and/or hot halo cooling), which in ETGs is expected to have large (super-solar) metal abundances. These and other observational and theoretical constraints analysed in previous works strongly support an external origin of the cold ISM in NGC 3100, probably via interaction with the companion gas-rich star-forming galaxy NGC 3095. Our finding also provides support to a scenario in which galaxy-galaxy interactions have a major role in replenishing the cold ISM reservoirs of LERGs located in poor environments, such as NGC 3100,

\section{ACKNOWLEDGEMENTS}

We thank the anonymous referee for useful comments that helped us improving the original manuscript. IR also thanks $\mathrm{Q}$. D'Amato for useful discussions. IR and TAD acknowledge support from the UK Science and Technology Facilities Council through grants ST/S00033X/1 and ST/W000830/1. IP acknowledges support from INAF under the SKA/CTA PRIN 'FORECaST' and from PRIN MIUR project 'Black Hole winds and the Baryon Life Cycle of Galaxies: the stone-guest at the galaxy evolution supper', contract \#2017PH3WAT. RP acknowledges the Italian PRIN-Miur 2017 (PI A.Cimatti). Part of this work has been carried out in the framework of the first author's $\mathrm{PhD}$ research project (IR PhD Thesis: http://amsdottorato.unibo.it/9244 /1/PhDThesis_IRuffa_finalversion.pdf). This paper makes use of the following ALMA data: ADS/JAO.ALMA\#[2015.1.01572.S] and ADS/JAO.ALMA\#[2018.1.01095.S]. ALMA is a partnership of ESO (representing its member states), NSF (USA) and NINS (Japan), together with NRC (Canada), NSC and ASIAA (Taiwan), and KASI (Republic of Korea), in cooperation with the Republic of Chile. The Joint ALMA Observatory is operated by ESO, AUI/NRAO and
NAOJ. The National Radio Astronomy Observatory is a facility of the National Science Foundation operated under cooperative agreement by Associated Universities, Inc. This paper has also made use of the NASA/IPAC Extragalactic Database (NED), which is operated by the Jet Propulsion Laboratory, California Institute of Technology under contract with NASA. This research used the facilities of the Canadian Astronomy Data Centre operated by the National Research Council of Canada with the support of the Canadian Space Agency. This research made use of Astropy, a community-developed core Python package for Astronomy (Astropy Collaboration 2013). We acknowledge the usage of the HyperLeda data base (http://leda.univ-lyon1.fr).

\section{DATA AVAILABILITY}

The ALMA data used in this article are available to download at the ALMA archive (https://almascience.nrao.edu/asax/; project codes: 2015.1.01572.S and 2018.1.01095.S). The calibrated data, final data products, and original plots generated for the research study underlying this article will be shared upon reasonable request to the first author.

\section{REFERENCES}

Alatalo K. et al., 2013, MNRAS, 432, 1796

Asplund M., Grevesse N., Sauval A. J., Allende Prieto C., Kiselman D., 2004, A\&A, 417, 751

Astropy Collaboration, 2013, A\&A, 558, A33

Best P. N., Heckman T. M., 2012, MNRAS, 421, 1569

Bieri R., Dubois Y., Rosdahl J., Wagner A., Silk J., Mamon G. A., 2017, MNRAS, 464, 1854

Bigiel F. et al., 2016, ApJ, 822, L26

Bolatto A. D., Wolfire M., Leroy A. K., 2013, ARA\&A, 51, 207

Bosma A., 1981a, AJ, 86, 1791

Bosma A., 1981b, AJ, 86, 1825

Brown M. J. I., Jannuzi B. T., Floyd D. J. E., Mould J. R., 2011, ApJ, 731, L41

Carilli C. L., Walter F., 2013, ARA\&A, 51, 105

Casasola V. et al., 2020, A\&A, 633, A100

Cavagnolo K. W., McNamara B. R., Nulsen P. E. J., Carilli C. L., Jones C., Bîrzan L., 2010, ApJ, 720, 1066

Choi E., Somerville R. S., Ostriker J. P., Naab T., Hirschmann M., 2018, ApJ, 866,91

Cielo S., Bieri R., Volonteri M., Wagner A. Y., Dubois Y., 2018, MNRAS, 477, 1336

Colless M. et al., 2003, preprint (arXiv:astro-ph/0306581)

Collobert M., Sarzi M., Davies R. L., Kuntschner H., Colless M., 2006, MNRAS, 370, 1213

Combes F., 2017, Front. Astron. Space Sci., 4, 10

Combes F. et al., 2013, A\&A, 558, A124

Cormier D. et al., 2018, MNRAS, 475, 3909

Costagliola F. et al., 2011, A\&A, 528, A30

Dame T. M., 2011, preprint (arXiv:1101.1499)

Dasyra K. M., Combes F., Oosterloo T., Oonk J. B. R., Morganti R., Salomé P., Vlahakis N., 2016, A\&A, 595, L7

Davis T. A., Bureau M., 2016, MNRAS, 457, 272

Davis T. A. et al., 2013, MNRAS, 429, 534

Davis T. A. et al., 2015, MNRAS, 454, 657

Davis T. A., Bureau M., Onishi K., Cappellari M., Iguchi S., Sarzi M., 2017, MNRAS, 468, 4675

Davis T. A., Greene J. E., Ma C.-P., Blakeslee J. P., Dawson J. M., Pandya V., Veale M., Zabel N., 2019, MNRAS, 486, 1404

De Vaucouleurs G., 1976, in Dickens R. J., Perry J. E., Smith F. G., King I. R., eds, Royal Greenwich Observatory Bulletins, Vol. 182, The Galaxy and the Local Group, p. 177

Domínguez-Fernández A. J. et al., 2020, A\&A, 643, A127 
Dopita M. A. et al., 2015, ApJS, 217, 12

Draine B. T. et al., 2007, ApJ, 663, 866

Ekers R. D. et al., 1989, MNRAS, 236, 737

Fanaroff B. L., Riley J. M., 1974, MNRAS, 167, 31P

Fathi K. et al., 2013, ApJ, 770, L27

Feruglio C. et al., 2015, A\&A, 583, A99

Finkelman I. et al., 2008, MNRAS, 390, 969

Gao Y., Solomon P. M., 2004, ApJ, 606, 271

García-Burillo S. et al., 2010, A\&A, 519, A2

García-Burillo S. et al., 2014, A\&A, 567, A125

Gaspari M., Ruszkowski M., Oh S. P., 2013, MNRAS, 432, 3401

Godfrey L. E. H., Shabala S. S., 2013, ApJ, 767, 12

Godfrey L. E. H., Shabala S. S., 2016, MNRAS, 456, 1172

Greve T. R., Papadopoulos P. P., Gao Y., Radford S. J. E., 2009, ApJ, 692, 1432

Hardcastle M., 2018, Nature Astron., 2, 273

Hardcastle M. J., Evans D. A., Croston J. H., 2007, MNRAS, 376, 1849

Harrison C. M., Molyneux S. J., Scholtz J., Jarvis M. E., 2021, in Storchi Bergmann T., Forman W., Overzier R., Riffel R., eds, Galaxy Evolution and Feedback across Different Environments, Vol. 359, Cambridge Univ. Press, Cambridge, p. 203

Heckman T. M., Kauffmann G., Brinchmann J., Charlot S., Tremonti C., White S. D. M., 2004, ApJ, 613, 109

Hirashita H., Nozawa T., 2017, Planet. Space Sci., 149, 45

Ho L. C., Li Z.-Y., Barth A. J., Seigar M. S., Peng C. Y., 2011, ApJS, 197, 21

Hopkins P. F., Quataert E., 2010, MNRAS, 407, 1529

Ishibashi W., Fabian A. C., Maiolino R., 2018, MNRAS, 476, 512

Jiménez-Donaire M. J. et al., 2017, ApJ, 836, L29

Jones D. H. et al., 2009, MNRAS, 399, 683

Kamenetzky J., Rangwala N., Glenn J., 2017, MNRAS, 471, 2917

Kamenetzky J., Privon G. C., Narayanan D., 2018, ApJ, 859, 9

Kaviraj S. et al., 2012, MNRAS, 423, 49

Kelly G., Viti S., García-Burillo S., Fuente A., Usero A., Krips M., Neri R., 2017, A\&A, 597, A11

Kerr F. J., Lynden-Bell D., 1986, MNRAS, 221, 1023

King A., Nixon C., 2015, MNRAS, 453, L46

Koay J. Y., Vestergaard M., Casasola V., Lawther D., Peterson B. M., 2016, MNRAS, 455, 2745

König S. et al., 2018, A\&A, 615, A122

Lagos C. d. P., Padilla N. D., Davis T. A., Lacey C. G., Baugh C. M., GonzalezPerez V., Zwaan M. A., Contreras S., 2015, MNRAS, 448, 1271

Laing R. A., Bridle A. H., 2013, MNRAS, 432, 1114

Laurikainen E., Salo H., Buta R., Knapen J., Speltincx T., Block D., 2006, AJ, 132, 2634

Lim J., Ao Y., Dinh-V-Trung, 2008, ApJ, 672, 252

McMullin J. P., Waters B., Schiebel D., Young W., Golap K., 2007, in Shaw R. A., Hill F., Bell D. J., eds, ASP Conf. Ser. Vol. 376, Astronomical Data Analysis Software and Systems XVI. Astron. Soc. Pac., San Francisco, p. 127

McNamara B. R., Nulsen P. E. J., 2012, New J. Phys., 14, 055023

Mahony E. K., Oonk J. B. R., Morganti R., Tadhunter C., Bessiere P., Short P., Emonts B. H. C., Oosterloo T. A., 2016, MNRAS, 455, 2453

Maiolino R. et al., 2012, MNRAS, 425, L66

Martini P., Dicken D., Storchi-Bergmann T., 2013, ApJ, 766, 121

Mathews W. G., Brighenti F., 2003, ARA\&A, 41, 191

Morganti R., 2017, Front. Astron. Space Sci., 4, 42

Morganti R., Oosterloo T., Oonk J. B. R., Frieswijk W., Tadhunter C., 2015, A\&A, 580, A1

Morganti R., Oosterloo T., Tadhunter C. N., 2021, in Storchi Bergmann T., Forman W., Overzier R., Riffel R., eds, Galaxy Evolution and Feedback across Different Environments, Vol. 359, Cambridge Univ. Press, Cambridge, p. 243

Mukherjee D., Bicknell G. V., Sutherland R., Wagner A., 2016, MNRAS, 461,967

Mukherjee D., Wagner A. Y., Bicknell G. V., Morganti R., Oosterloo T., Nesvadba N., Sutherland R. S., 2018a, MNRAS, 476, 80

Mukherjee D., Bicknell G. V., Wagner A. Y., Sutherland R. S., Silk J., 2018b, MNRAS, 479, 5544
Mukherjee D., Bicknell G. V., Wagner A. Y., 2021, preprint (arXiv:2110.11900)

Murthy S. et al., 2019, A\&A, 629, A58

Narayan R., Quataert E., Igumenshchev I. V., Abramowicz M. A., 2002, ApJ, 577,295

Nayakshin S., Power C., King A. R., 2012, ApJ, 753, 15

Newville M., Stensitzki T., Allen D. B., Ingargiola A., 2014, LMFIT: Non-Linear Least-Square Minimization and Curve-Fitting for Python. Zenodo. Available at: https://doi.org/10.5281/zenodo.11813

O’Dea C. P., Saikia D. J., 2021, A\&AR, 29, 3

Oosterloo T., Raymond Oonk J. B., Morganti R., Combes F., Dasyra K., Salomé P., Vlahakis N., Tadhunter C., 2017, A\&A, 608, A38

Petrov L., Kovalev Y. Y., Fomalont E., Gordon D., 2005, AJ, 129, 1163

Pillepich A. et al., 2019, MNRAS, 490, 3196

Rau U., Cornwell T. J., 2011, A\&A, 532, A71

Ruffa I. et al., 2019a, MNRAS, 484, 4239 (Paper I)

Ruffa I. et al., 2019b, MNRAS, 489, 3739 (Paper II)

Ruffa I., Laing R. A., Prandoni I., Paladino R., Parma P., Davis T. A., Bureau M., 2020, MNRAS, 499, 5719 (Paper III)

Rupen M. P., 1999, in Taylor G. B., Carilli C. L., Perley R. A., eds, ASP Conf. Ser. Vol. 180, Synthesis Imaging in Radio Astronomy II. Astron. Soc. Pac., San Francisco, p. 229

Russell H. R. et al., 2016, MNRAS, 458, 3134

Sabater J., Best P. N., Heckman T. M., 2015, MNRAS, 447, 110

Saito T. et al., 2017, ApJ, 835, 174

Sandage A., Brucato R., 1979, AJ, 84, 472

Santoro F., Tadhunter C., Baron D., Morganti R., Holt J., 2020, A\&A, 644, A54

Schilke P., Walmsley C. M., Pineau des Forets G., Flower D. R., 1997, A\&A, 321, 293

Smith R. J., Lucey J. R., Hudson M. J., Schlegel D. J., Davies R. L., 2000, MNRAS, 313, 469

Storchi-Bergmann T., Schnorr-Müller A., 2019, Nature Astron., 3, 48

Swinbank A. M., Sobral D., Smail I., Geach J. E., Best P. N., McCarthy I. G., Crain R. A., Theuns T., 2012, MNRAS, 426, 935

Tadhunter C. N., Morganti R., di Serego-Alighieri S., Fosbury R. A. E., Danziger I. J., 1993, MNRAS, 263, 999

Temi P., Amblard A., Gitti M., Brighenti F., Gaspari M., Mathews W. G., David L., 2018, ApJ, 858, 17

Topal S., Bureau M., Davis T. A., Krips M., Young L. M., Crocker A. F., 2016, MNRAS, 463, 4121

Tremaine S. et al., 2002, ApJ, 574, 740

Tremblay G. R. et al., 2016, Nature, 534, 218

Usero A., García-Burillo S., Martín-Pintado J., Fuente A., Neri R., 2006, A\&A, 448, 457

Usero A. et al., 2015, AJ, 150, 115

Utomo D., Blitz L., Davis T., Rosolowsky E., Bureau M., Cappellari M., Sarzi M., 2015, ApJ, 803, 16

Valentini M., Brighenti F., 2015, MNRAS, 448, 1979

van de Ven G., Fathi K., 2010, ApJ, 723, 767

van de Voort F. et al., 2018, MNRAS, 476, 122

van der Kruit P. C., Shostak G. S., 1982, A\&A, 105, 351

Veilleux S., Maiolino R., Bolatto A. D., Aalto S., 2020, A\&AR, 28, 2

Venturi G. et al., 2021, A\&A, 648, A17

Wagner A. Y., Bicknell G. V., Umemura M., 2012, ApJ, 757, 136

Wagner A. Y., Bicknell G. V., Umemura M., Sutherland R. S., Silk J., 2016, Astron. Nachr., 337, 167

Wrobel J. M., Walker R. C., 1999, in Taylor G. B., Carilli C. L., Perley R. A., eds, ASP Conf. Ser. Vol. 180, Synthesis Imaging in Radio Astronomy II. Astron. Soc. Pac., San Francisco, p. 171

Wylezalek D., Morganti R., 2018, Nature Astron., 2, 181

Young L. M. et al., 2014, MNRAS, 444, 3408

Young L. M., Meier D. S., Bureau M., Crocker A., Davis T. A., Topal S., 2021, ApJ, 909, 98

Zovaro H. R. M., Sharp R., Nesvadba N. P. H., Bicknell G. V., Mukherjee D., Wagner A. Y., Groves B., Krishna S., 2019, MNRAS, 484, 3393

This paper has been typeset from a $\mathrm{T}_{\mathrm{E}} \mathrm{X} / \mathrm{L} \mathrm{T} \mathrm{E} \mathrm{X}$ file prepared by the author. 